\title{
REGULARITY OF CENTRAL LEAVES OF PARTIALLY HYPERBOLIC SETS AND ITS APPLICATIONS
}

\author{
A.Gorodetski
}

\section{$\S 1$. INTRODUCTION}

Consider a map $S: N \rightarrow N$ of a manifold $N$ which has a locally maximal hyperbolic set $\Lambda \subset N$. Let $M$ be a closed smooth manifold. Properties of small perturbations of the map $S \times i d_{M}: N \times M \rightarrow N \times M$ were studied in many different ways (Hirsh-Pugh-Shub [13], Shub-Wilkinson [25], Ruelle-Wilkinson [22], Nitica-Török [19], Bonatti-Diaz [3], see also Pesin [20]). In this paper we establish the Hölder dependence of central leaves of a perturbed map on the point in base in $C^{r}$-metric. Then we use this result to complete the proof of the following theorem that was announced by Yu.Ilyashenko and the author in [9], [10].

Theorem A. Given an open finite interval $I \subset \mathbb{R}, 0 \in I$ and a closed manifold $M, \operatorname{dim} M \geq 3$, there exists an open set $U \subset$ Diff $^{2}(M)$ such that any $f \in U$ has a locally maximal invariant set $\Delta \subset M$ with the following properties:

(i) There exist two numbers $l_{1}$ and $l_{2}=l_{1}+1$ such that the hyperbolic periodic orbits with stable manifolds of dimension $l_{i}$ are dense in $\Delta$,

(ii) For any $\lambda \in I$ there exists an orbit dense in $\Delta$ with one of the intermediate Lyapunov exponents equal to $\lambda$.

Addendum. The set $\Delta$ in Theorem $A$ may for $\operatorname{dim} M \geq 4$ be taken to be a partially hyperbolic attractor.

The property $(i)$ has some further consequences (see [8], [17]). Using different ideas the property $(i)$ for some partially hyperbolic diffeomorphisms which are close to a direct product of a hyperbolic map by identity was proved by C.Bonatti and L.J.Diaz [3].

The proof of Theorem A is based on properties of skew products over horseshoe and solenoid [10] and the following theorem B.

Theorem B. Let the map $\mathfrak{F}: N \times M \rightarrow N \times M, \mathfrak{F}=S \times i d_{M}$ be of class $C^{r+1}, 0 \leq r<\infty$. Then for any $C^{r+1}$-diffeomorphism $\mathfrak{G}$, which is $C^{r+1}$-close to $\mathfrak{F}$, there is an invariant subset $\Delta$ and a homeomorphism $\Psi: \Lambda \times M \rightarrow \Delta$. Moreover,

The author was partially supported by the grants RFBR 02-01-00482 and 02-01-22002. 
if $p_{1}: \Lambda \times M \rightarrow \Lambda$ is a projection to the first factor, then the map $\Phi=p_{1} \circ \Psi^{-1}$, $\Phi:(\Delta, \mathfrak{G}) \rightarrow(\Lambda, S)$ is a semiconjugacy, the leaves $\Phi^{-1}(x)$ are $C^{r+1}$-smooth and depend Hölder continuously on a point $x \in \Lambda$ in $C^{r}$-metric. The Hölder exponent and the Hölder constant are uniform on a small $C^{r+1}$-neighborhood of the map $\mathfrak{F}$.

For the case when $S$ is an Anosov diffeomorphism of $\mathbb{T}^{n}$ and $M=\mathbb{T}^{k}$ this result (by different method) was received by V.Nitica and A.Török [18].

Numerous other results about Hölder structures related to uniform and partial hyperbolicity were obtained by Anosov [2], Brin [6], Brin-Pesin [7], Pugh-ShubWilkinson [21], Hasselbladt [12], Schmeling and Siegmund-Schultze [23].

Recently V.Kleptsyn and M.Nalsky [16], based on [11], used Theorem B to construct $C^{1}$-open set of diffeomorphisms with non-hyperbolic invariant ergodic measures.

The organization of the paper is as follows. In $\S 2$ we recall the notion of intrinsically hyperbolic map and prove that the conjugacy of two intrinsically hyperbolic maps has to be Hölder continuous. In $\S 3$ we consider a small perturbation of a direct product of hyperbolic map by identity, the space of its central leaves equipped with $C^{r}$-metric and prove that the induced map on this space is intrinsically hyperbolic. Together with result of $\S 2$ (Theorem 2.2 and 2.3) it immediately gives Theorem B. Finally, in $\S 4$ we apply Theorem B and results concerning a properties of skew products of a special kind obtained by Yu.Ilyashenko and the author in [10] to study the properties of certain partially hyperbolic maps. It allows to get the proof of Theorem A.

\section{§2. INTRINSICALLY HYPERBOLIC HOMEOMORPHISMS}

Here we prove Theorem 2.2 (analog of Theorem 5 in [9]) which will be used later to prove the Hölder dependence in $C^{r}$-metric of central leaves of partially hyperbolic invariant sets on the point in base. In section 2.1 some known facts about locally maximal hyperbolic sets are given. Section 2.2 contains the definition and some properties of intrinsically hyperbolic homeomorphisms (see [1], [5]). Finally, in section 2.3 we give the statement and the proof of the main result of this part, Theorem 2.2.

\subsection{Hölder continuity of conjugacies of hyperbolic sets.}

Definition 2.1. The invariant hyperbolic set $\Lambda$ of the map $S: N \rightarrow N$ is said to be locally maximal if there is a neighborhood $U$ of $\Lambda$ such that any $S$-invariant set in $U$, which contains $\Lambda$, coincides with $\Lambda$.

Let $\Lambda \subset N$ be a locally maximal hyperbolic set of the map $S: N \rightarrow N$. Let $d(\cdot, \cdot)$ be the metric on $N$.

Let us recall the standard notations:

$$
W^{u}(x)=\left\{y \mid d\left(S^{-n}(x), S^{-n}(y)\right) \underset{n \rightarrow+\infty}{\longrightarrow} 0\right\}
$$




$$
\begin{gathered}
W^{s}(x)=\left\{y \mid d\left(S^{n}(x), S^{n}(y)\right) \underset{n \rightarrow+\infty}{\longrightarrow} 0\right\}, \\
W_{\varepsilon}^{u}(x)=\left\{z \mid d\left(f^{-n}(x), f^{-n}(y)\right) \leq \varepsilon \text { for all } n \in \mathbb{Z}^{+}\right\}, \\
W_{\varepsilon}^{s}(x)=\left\{z \mid d\left(f^{n}(x), f^{n}(y)\right) \leq \varepsilon \text { for all } n \in \mathbb{Z}^{+}\right\} .
\end{gathered}
$$

Definition 2.2. The invariant hyperbolic set $\Lambda$ of the map $S: N \rightarrow N$ has local product structure if there exist $\varepsilon>0$ and $\delta>0$ such that the following holds. If $d(x, y)<\delta$ then the intersection $W_{\varepsilon}^{u}(x) \cap W_{\varepsilon}^{s}(y)$ consists of exactly one point, which belongs to $\Lambda$.

Proposition 2.1. [15, Theorem 18.4.1] The invariant hyperbolic set $\Lambda$ is locally maximal if and only if it has local product structure.

Theorem 2.1. [15, Theorem 19.1.2] Let $\Lambda$ and $\Lambda^{\prime}$ be locally maximal hyperbolic sets for diffeomorphisms $S$ and $S^{\prime}$, respectively, and $h: \Lambda \rightarrow \Lambda^{\prime}$ a topological conjugacy: $h=S^{\prime} h S^{-1}$. Then both $h$ and $h^{-1}$ are Hölder continuous.

Remark 2.1. Theorem 2.1 was originally proved by D.Anosov for Anosov diffeomorphisms (not published).

2.2. Intrinsically hyperbolic homeomorphisms: definition and main properties. Here we recall the notion of intrinsically hyperbolic homeomorphism (as an example one can take a restriction of a diffeomorphism to its locally maximal hyperbolic set). This object was introduced by Alekseev and Yakobson in [1, sec-

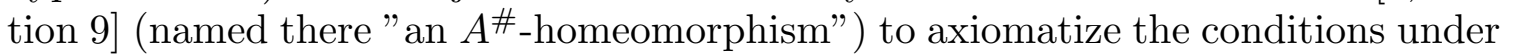
which the construction of the Markov partition and the symbolic dynamics is possible for a homeomorphism of a metric compactum. They modified an Axiom $A^{*}$ introduced by R.Bowen in [5]. Here this notion allows us to prove the generalization of Theorem 2.1 (namely, Theorem 2.2) and then use it in $\S 3$.

Let $(\Lambda, d)$ be a compact metric space, $S: \Lambda \rightarrow \Lambda$ be a lipschitzemorphism (i.e. $S$ is a homeomorphism, $S$ and $S^{-1}$ are Lipschitz continuous). Define the sets $W^{u}(x)$, $W^{s}(x), W_{\varepsilon}^{u}(x), W_{\varepsilon}^{s}(x)$ by formulas $(2.1)-(2.4)$.

Definition 2.3. A lipschitzemorphism $S$ of a compact metric space $(\Lambda, d)$ is intrinsically hyperbolic iff there exist $\delta>0$ and $\varepsilon>0$ such that the following hold:

(IH1) If $d(x, y)<\delta$ then there exists the only point $w=w(x, y) \in \Lambda$ such that $w \in W_{\varepsilon}^{u}(x) \cap W_{\varepsilon}^{s}(y)$.

(IH2) There exists a constant $0<\lambda<1$ such that

$$
\begin{gathered}
y \in W_{\varepsilon}^{s}(x) \Longrightarrow d\left(S^{n}(x), S^{n}(y)\right) \leq \lambda^{n} d(x, y), \quad n \in \mathbb{N}, \\
y \in W_{\varepsilon}^{u}(x) \Longrightarrow d\left(S^{-n}(x), S^{-n}(y)\right) \leq \lambda^{n} d(x, y), \quad n \in \mathbb{N} .
\end{gathered}
$$


The simplest examples are provided by locally maximal hyperbolic sets (with Lyapunov metric, see (3.1) and (3.1')) and the Bernoulli shifts.

Remark 2.2. Formally our definition is different from the definition of $A^{\# \text {-homeo- }}$ morphism by Alekseev and Yakobson, since we do not require the continuity of the map $(x, y) \rightarrow w(x, y)$ but require the Lipschitz condition for the map. Below we prove that the continuity of $w(x, y)$ follows from other conditions in our case.

Proposition 2.2. Let the map $S: \Lambda \rightarrow \Lambda$ be intrinsically hyperbolic. Then it is expansive with constant of expansivity $\varepsilon$, that is if $d\left(S^{n}(x), S^{n}(y)\right) \leq \varepsilon$ for some $x, y \in \Lambda$ and for all $n \in \mathbb{Z}$, then $x=y$.

Proof of Proposition 2.2. Since $d\left(S^{n}(x), S^{n}(y)\right) \leq \varepsilon$ for all $n \in \mathbb{Z}$ we have $y \in W_{\varepsilon}^{s}(x)$ and $y \in W_{\varepsilon}^{u}(x)$. Property (IH1) implies that $x=w(x, x)$ is the only point of intersection of $W_{\varepsilon}^{s}(x)$ and $W_{\varepsilon}^{u}(x)$, therefore $x=y$. Proposition 2.2 is proved.

Proposition 2.3. Let the map $S: \Lambda \rightarrow \Lambda$ be intrinsically hyperbolic. Take $\varepsilon, \delta$ and $\lambda$ as in definition 2.3. For any $x, y \in \Lambda$ and sequences $\left\{x_{n}\right\},\left\{y_{n}\right\} \subset \Lambda$ such that $d(x, y)<\delta$ and $x_{n} \rightarrow x, y_{n} \rightarrow y$ as $n \rightarrow+\infty$ we have $w\left(x_{n}, y_{n}\right) \rightarrow w(x, y)$, that is the map $w:(x, y) \mapsto W_{\varepsilon}^{u}(x) \cap W_{\varepsilon}^{s}(y)$ is continuous.

Proof of Proposition 2.3. Let us denote $w_{n}=w\left(x_{n}, y_{n}\right) \in \Lambda$. If $w_{n} \nrightarrow w(x, y)$ then by compactness of $\Lambda$ we can choose subsequence $w_{n_{k}}$ such that $\lim _{k \rightarrow \infty} w_{n_{k}}=w^{\prime} \neq$ $w(x, y)$. Let us show that $w^{\prime} \in W_{\varepsilon}^{s}(y)$. Indeed, $w_{n} \in W_{\varepsilon}^{s}\left(y_{n}\right) \Rightarrow d\left(y_{n}, w_{n}\right) \leq \varepsilon$, hence $\lim _{k \rightarrow \infty} d\left(y_{n_{k}}, w_{n_{k}}\right)=d\left(y, w^{\prime}\right) \leq \varepsilon$. Now let us show that for every $l \geq 1$ we also have $d\left(S^{l}(y), S^{l}\left(w^{\prime}\right)\right) \leq \varepsilon$. Let $P$ be a Lipschitz constant for $S$. Then there exists $N=N(l)$ such that $d\left(y, y_{N}\right)<\frac{1-\lambda^{l}}{2 P^{l}} \varepsilon$, and $d\left(w^{\prime}, w_{N}\right)<\frac{1-\lambda^{l}}{2 P^{l}} \varepsilon$. Since $d\left(S^{l}\left(y_{N}\right), S^{l}\left(w_{N}\right)\right) \leq \lambda^{l} d\left(y_{N}, w_{N}\right) \leq \lambda^{l} \varepsilon$, we have

$$
\begin{gathered}
d\left(S^{l}(y), S^{l}\left(w^{\prime}\right)\right) \leq d\left(S^{l}(y), S^{l}\left(y_{N}\right)\right)+d\left(S^{l}\left(y_{N}\right), S^{l}\left(w_{N}\right)\right)+d\left(S^{l}\left(w_{N}\right), S^{l}\left(w^{\prime}\right)\right) \leq \\
P^{l} \frac{1-\lambda^{l}}{2 P^{l}} \varepsilon+\lambda^{l} \varepsilon+P^{l} \frac{1-\lambda^{l}}{2 P^{l}} \varepsilon \leq \varepsilon .
\end{gathered}
$$

By definition this implies $w^{\prime} \in W_{\varepsilon}^{s}(y)$.

In a similar way we have $w^{\prime} \in W_{\varepsilon}^{u}(x)$. But by (IH1) there exists only one point in intersection $W_{\varepsilon}^{u}(x) \cap W_{\varepsilon}^{s}(y)$, therefore $w^{\prime}=w(x, y)$. Proposition 2.3 is proved.

\subsection{Hölder continuity of conjugacies: intrinsically hyperbolic case.}

Theorem 2.2. Let $\Lambda$ and $\Lambda^{\prime}$ be compact metric spaces, lipschitzemorphisms $S$ : $\Lambda \rightarrow \Lambda$ and $S^{\prime}: \Lambda^{\prime} \rightarrow \Lambda^{\prime}$ are intrinsically hyperbolic and $h: \Lambda \rightarrow \Lambda^{\prime}$ be a topological conjugacy: $h=S^{\prime} h S^{-1}$. Then both $h$ and $h^{-1}$ are Hölder continuous.

We need also to be able to estimate the Hölder exponent of the obtained conjugacy. This is provided by the following statement: 
Theorem 2.3. Let $\Lambda$ and $\Lambda^{\prime}$ be compact metric spaces, lipschitzemorphisms $S$ : $\Lambda \rightarrow \Lambda$ and $S^{\prime}: \Lambda^{\prime} \rightarrow \Lambda^{\prime}$ are intrinsically hyperbolic with constants $\lambda$ and $\lambda^{\prime}$, respectively, and $h: \Lambda \rightarrow \Lambda^{\prime}$ a topological conjugacy: $h=S^{\prime} h S^{-1}$.

Let $P$ be a Lipschitz constant for $S$ and $Q$ a Lipschitz constant for $S^{-1}$.

Let for some $\alpha>0, \alpha^{\prime}>0$ the inequalities hold:

$$
\lambda P^{\alpha}<1, \quad \lambda Q^{\alpha}<1, \quad \lambda^{\prime} P^{\alpha^{\prime}}<1, \quad \lambda^{\prime} Q^{\alpha^{\prime}}<1 .
$$

Then the conjugacy $h$ is Hölder continuous with the Hölder exponent equal to $\alpha \alpha^{\prime}$.

Proof of Theorem 2.3. The proof of Theorem 2.3 is similar to the proof of classical Theorem 2.1 (we follow the ideas of [15, Chapter 19]). The only problem is that we can not use the uniform transversality of stable and unstable manifolds. So we use Lemma 2.2 instead.

Lemma 2.1. Let $\alpha>0$. There exists a constant $K>0, K=K(\alpha)$, such that for any $a>0, b>0$ the following inequality holds:

$$
\left(a^{\alpha}+b^{\alpha}\right)^{1 / \alpha} \leq K(a+b)
$$

Proof of Lemma 2.1. For any $\alpha>0$ the function $\frac{\left(1+x^{\alpha}\right)^{1 / \alpha}}{1+x}$ is bounded when $x \geq 0$. It gives the required inequality if one sets $x=\frac{b}{a}$.

Lemma 2.2. Let the map $S: \Lambda \rightarrow \Lambda$ be intrinsically hyperbolic with constant $\lambda$. Let $P$ be a Lipschitz constant for $S$ and $Q$ a Lipschitz constant for $S^{-1}$. Let for some $\alpha>0$ the inequalities hold: $\lambda P^{\alpha}<1, \lambda Q^{\alpha}<1$. Then there exists $L>1$ such that if $w=w(x, y)=W_{\varepsilon}^{u}(x) \cap W_{\varepsilon}^{s}(y)$ then

$$
d(x, w)+d(w, y) \leq L d^{\alpha}(x, y) .
$$

Proof of Lemma 2.2. By Proposition $2.2 \varepsilon$ is an expansivity constant for the map $S$. There exits $L_{1} \geq 1$ such that if $d(x, y)>\varepsilon$ then $\frac{d(x, w)+d(w, y)}{d^{\alpha}(x, y)} \leq L_{1}$. Let us show that the statement of Lemma 2.2 holds for $L=2 L_{1}$.

If $w(x, y)=x$ or $w(x, y)=y$ then there is nothing to prove. Otherwise we have $d\left(S^{k}(x), S^{k}(y)\right) \geq \varepsilon$ for certain $k \in \mathbb{N}$. Let us estimate $d(x, w)$ using (IH2) and inequality $\lambda P^{\alpha} \leq 1$. We have: $d(x, w) \leq \lambda^{k} d\left(S^{k}(x), S^{k}(w)\right) \leq$ $\lambda^{k} L_{1} d^{\alpha}\left(S^{k}(x), S^{k}(y)\right) \leq \lambda^{k} L_{1} P^{k} \alpha d^{\alpha}(x, y) \leq L_{1} d^{\alpha}(x, y)$. Analogously $d(w, y) \leq$ $L_{1} d^{\alpha}(x, y)$.

Therefore $d(x, w)+d(w, y) \leq 2 L_{1} d^{\alpha}(x, y) \leq L d^{\alpha}(x, y)$. Lemma 2.2 is proved.

Definition 2.3. Let $\Lambda, \Lambda^{\prime}$ be metric spaces. The map $h: \Lambda \rightarrow \Lambda$ is called locally Hölder with exponent $\alpha$ if there exist $\gamma>0$ and $C>0$ such that

$$
d_{\Lambda}(x, y)<\gamma \quad \Longrightarrow \quad d_{\Lambda^{\prime}}(h(x), h(y)) \leq C d_{\Lambda}^{\alpha}(x, y) .
$$


Proposition 2.4. Let $\Lambda, \Lambda^{\prime}$ be compact metric spaces. Let the map $h: \Lambda \rightarrow \Lambda$ be locally Hölder with exponent $\alpha$. Then it is Hölder continuous with exponent $\alpha$.

Remark. In Proposition 2.4 the exponent $\alpha$ can be greater than 1 .

Proof of Proposition 2.4. By Definition 2.3 there exist $\gamma>0$ and $C>0$ such that if $d_{\Lambda}(x, y)<\gamma$ then $d_{\Lambda^{\prime}}(h(x), h(y)) \leq C d_{\Lambda}^{\alpha}(x, y)$. Let $R=\max \left(C, \gamma^{-\alpha} \operatorname{diam} \Lambda^{\prime}\right)$.

Now if $d_{\Lambda}(x, y)<\gamma$ then $d_{\Lambda^{\prime}}(h(x), h(y)) \leq C d_{\Lambda}^{\alpha}(x, y) \leq R d_{\Lambda}^{\alpha}(x, y)$. But if $d_{\Lambda}(x, y) \geq \gamma$ then $d_{\Lambda^{\prime}}(h(x), h(y)) \leq \operatorname{diam} \Lambda^{\prime} \leq\left(\gamma^{-\alpha} \operatorname{diam} \Lambda^{\prime}\right) \gamma^{\alpha} \leq R d_{\Lambda}^{\alpha}(x, y)$. Therefore the map $h$ is Hölder continuous with exponent $\alpha$ (and constant $R$ ).

Lemma 2.3. Let the conditions of Theorem 2.3 hold. Assume that there exists $K_{1}>0$ such that for any $x, y \in \Lambda, y \in W_{\gamma}^{u}(x)$ or $y \in W_{\gamma}^{s}(x)$, the inequality holds: $d(h(x), h(y)) \leq K_{1} d^{\alpha^{\prime}}(x, y)$. Then the map $h$ is locally Hölder with exponent $\alpha \alpha^{\prime}$.

Proof of Lemma 2.3. By Lemma 2.1 and Lemma 2.2 we have: $d(h(x), h(y)) \leq$ $d(h(x), h(w))+d(h(w), h(y)) \leq K_{1}\left(d^{\alpha^{\prime}}(x, w)+d^{\alpha^{\prime}}(w, y)\right) \leq K^{\alpha^{\prime}} K_{1}(d(x, w)+$ $d(w, y))^{\alpha^{\prime}} \leq K^{\alpha^{\prime}} K_{1} L^{\alpha^{\prime}} d^{\alpha \alpha^{\prime}}(x, y)$. Lemma 2.3 is proved.

Now let us prove Theorem 2.3. It is enough to show that $h$ is Hölder continuous separately along $W_{\varepsilon}^{u}$ and along $W_{\varepsilon}^{s}$. Indeed, by Lemma 2.3 it implies that the map $h$ is locally Hölder continuous with exponent $\alpha \alpha^{\prime}$, and by Proposition 2.3 it is equivalent to a Hölder continuity with the same exponent.

Since $\Lambda$ is compact the map $h$ is uniformly continuous. Let us fix $\varepsilon_{0}>0$. There exists $\delta_{0}>0$ such that if $d(x, y)<\delta_{0}$ then $d(h(x), h(y))<\varepsilon_{0}$.

Note that (2.1) and (2.2) imply that if $x \in W^{u}(y)$ then $h(x) \in W^{u}(h(y))$, and if $x \in W^{s}(y)$ then $h(x) \in W^{s}(h(y))$.

Now let $x \in W^{u}(y)$ and $d(x, y)=d<\delta_{0}$. There exists $n \in \mathbb{N}$ such that $d\left(S^{n}(x), S^{n}(y)\right) \leq P^{n} d<\delta_{0} \leq P^{n+1} d$. Hence $d\left(h\left(S^{n}(x)\right), h\left(S^{n}(y)\right)\right)<\varepsilon_{0}$ and using inequality $\lambda^{\prime} P^{\alpha^{\prime}}<1$ we have: $d(h(x), h(y))=d\left(S^{\prime-n} h\left(S^{n}(x)\right), S^{\prime-n} h\left(S^{n}(y)\right)\right) \leq$ $\lambda^{\prime n} \varepsilon_{0}=\lambda^{\prime n} \delta_{0}^{\alpha^{\prime}}\left(\varepsilon_{0} / \delta_{0}^{\alpha^{\prime}}\right) \leq\left(\lambda^{\prime} P^{\alpha^{\prime}}\right)^{n} P^{\alpha^{\prime}}\left(\varepsilon_{0} / \delta_{0}^{\alpha^{\prime}}\right) d^{\alpha^{\prime}}<\left[P^{\alpha^{\prime}}\left(\varepsilon_{0} / \delta_{0}^{\alpha^{\prime}}\right)\right] d^{\alpha^{\prime}}(x, y)$.

The proof of the Hölder continuity of $h$ along $W_{\varepsilon}^{s}$ is similar (one has to note that $h$ conjugates $S^{-1}$ and $S^{\prime-1}$, and to use the inequality $\lambda^{\prime} Q^{\alpha^{\prime}}<1$ ). Theorem 2.3 is proved.

\section{§3. INTRINSIC HYPERBOLICITY OF THE INDUCED MAP ON THE SPACE OF CENTRAL LEAVES EQUIPPED WITH $C^{r}$-METRIC}

In this section we prove Theorem B. In order to do this we are going to consider the set of all central leaves as a metric space. Every single leaf is a point, and the metric is given by the $C^{r}$-distance between leaves. In this case the induced map defined below appears to be intrinsically hyperbolic (Theorem 3.2 ) and the application of Theorem 2.3 proves Theorem $B$.

\subsection{Smooth perturbations of direct products and their invariant sets.}

Let the map $S: N \rightarrow N$ have locally maximal hyperbolic set $\Lambda \subset N$. Let $U \subset N$ be a neighborhood of $\Lambda$ such that $\Lambda=\cap_{n \in \mathbb{Z}} S^{n}(U)$. Let $M$ be a closed manifold. 
The following statement is a particular case of Theorems 6.1, 6.8 and 7.1 from $[13]$.

Theorem 3.1. Let the map $\mathfrak{F}: N \times M \rightarrow N \times M,(x, \varphi) \mapsto(S(x), \varphi)$ be of class $C^{r+1}, 0 \leq r<\infty$. Then for any $C^{r+1}$-diffeomorphism $\mathfrak{G}$, there is an invariant set $\Delta \subset N \times M$ and a homeomorphism $\Psi: \Lambda \times M \rightarrow \Delta$. If $p_{1}: \Lambda \times M \rightarrow \Lambda$ is a projection to the first factor, then the map $\Phi=p_{1} \circ \Psi^{-1}, \Phi:(\Delta, \mathfrak{G}) \rightarrow(\Lambda, S)$ is a semiconjugacy. The central leaves $\Phi^{-1}(x), x \in \Lambda$, are $C^{r+1}$-smooth and $C^{r+1}$-tend to central leaves of $\mathfrak{F}$ (i.e. $\{x\} \times M)$ as $\mathfrak{G}$ tends to $\mathfrak{F}$ in $C^{r+1}$-topology. Local stable and unstable manifolds of central leaves of $\mathfrak{G}$ as well as local strong stable and strong unstable manifolds $C^{r+1}$-tend to the corresponding manifolds of $\mathfrak{F}$ as $\mathfrak{G}$ tends to $\mathfrak{F}$ in $C^{r+1}$-topology.

We want to prove the following generalization.

Theorem B. Under the conditions of Theorem 3.1 the central leaves $\Phi^{-1}(x)$ of a $C^{r+1}$-diffeomorphism $\mathfrak{G}$ which is $C^{r+1}$-close to $\mathfrak{F}$ depend Hölder continuously on a point $x \in \Lambda$ in $C^{r}$-metric. The Hölder exponent and the Hölder constant are uniform on a small $C^{r+1}$-neighborhood of the map $\mathfrak{F}$.

Let $\mathfrak{L}^{r}$ denote the metric space of all central leaves of the map $\mathfrak{G}$ with $C^{r}$-metric (which is defined below). A single leaf $\Phi^{-1}(x)$ is one point in $\mathfrak{L}^{r}$. Let us note that $\mathfrak{G}$ sends leaf to the leaf, so $\mathfrak{G}$ induces a well defined map $\mathfrak{g}: \mathfrak{L}^{r} \rightarrow \mathfrak{L}^{r}$. This is the induced map that appears in the title of $\S 3$. To prove Theorem $\mathrm{B}$ we show that the map $\mathfrak{g}$ is intrinsically hyperbolic. Also we show that the systems $\left(\Lambda,\left.S\right|_{\Lambda}\right)$ and $\left(\mathfrak{L}^{r}, \mathfrak{g}\right)$ are conjugate. The application of Theorem 2.3 directly gives the Theorem B.

Theorem 3.2. Let the map $S: N \rightarrow N$ have locally maximal hyperbolic set with constant $\lambda$ (i.e. (2.5) and (2.6) hold). Let the map $\mathfrak{F}: N \times M \rightarrow N \times M$, $(x, \varphi) \mapsto(S(x), \varphi)$ have class of smoothness $C^{r+1}, 1 \leq r<\infty$. Let $\lambda^{\prime}>\lambda$. Then for any $C^{r+1}$-diffeomorphism $\mathfrak{G}$, which is $C^{r+1}$-close to $\mathfrak{F}$, the map $\mathfrak{g}: \mathfrak{L}^{r} \rightarrow \mathfrak{L}^{r}$ induced on the space of central leaves is intrinsically hyperbolic with the constant of contraction $\lambda^{\prime}$ and the systems $\left(\Lambda,\left.S\right|_{\Lambda}\right)$ and $\left(\mathfrak{L}^{r}, \mathfrak{g}\right)$ are conjugate.

We prove Theorem 3.2 by induction in $r$.

3.2. Base of induction: $C^{0}$-metric. The map $S: N \rightarrow N$ has a locally maximal hyperbolic set $\Lambda$. In a small neighborhood $U(\Lambda)$ there exists a splitting $T_{U(\Lambda)} N=E^{s} \oplus E^{u}$ such that along stable distribution the differential of the map $S$ is contracting, and along unstable distribution its inverse is contracting. Assume that in a neighborhood $U(\Lambda)$ a Lyapunov metric is given, that is

$$
\begin{gathered}
\left\|D S_{x}(v)\right\| \leq \lambda\|v\|, \quad \text { if } \quad v \in E^{s}(x), x \in \Lambda, \\
\left\|D S_{x}^{-1}(v)\right\| \leq \lambda\|v\|, \quad \text { if } \quad v \in E^{u}(x), x \in \Lambda .
\end{gathered}
$$


Let the map $\mathfrak{G}$ be $C^{1}$-close to $\mathfrak{F}=S \times i d_{M}$. Let us denote by $\bar{x}$ a central leaf of the map $\mathfrak{G}$ over the point $x \in \Lambda$, that is $\bar{x}=\Phi^{-1}(x)$ (we will use this notation for a set $\Phi^{-1}(x)$ as well as for a point in $\left.\mathfrak{L}\right)$.

A central leaf $\bar{x}$ is $C^{1}$-close to the corresponding central leaf $\{x\} \times M$ of the map $\mathfrak{F}$, and therefore can be considered as a graph of a map $\hat{x}: M \rightarrow N$. Let us define $C^{0}$-distance between central leaves (i.e. $C^{0}$-metric in $\mathfrak{L}$ ) in the following way:

$$
d_{C^{0}}(\bar{x}, \bar{y})=\max _{\varphi \in M} d_{N}(\hat{x}(\varphi), \hat{y}(\varphi))
$$

where $d_{N}(\cdot, \cdot)$ is a metric on $N$.

In order to prove Theorem 3.2 for $r=0$ we need to show that the induced map $\mathfrak{g}: \mathfrak{L}^{0} \rightarrow \mathfrak{L}^{0}$ is a Lipschitzemorphism that satisfies the conditions (IH1) and (IH2) from Definition 2.2, and that the systems $\left(\Lambda,\left.S\right|_{\Lambda}\right)$ and $\left(\mathfrak{L}^{0}, \mathfrak{g}\right)$ are conjugate. Fix small $\rho>0$. Let us show first that $\mathfrak{g}$ satisfies the condition (IH2) if $\mathfrak{G}$ is $C^{1}$-close enough to $\mathfrak{F}$.

\section{Lemma 3.1.}

If $x \in W_{l o c}^{s}(y)$ then $d_{C^{0}}(\mathfrak{g} \bar{x}, \mathfrak{g} \bar{y}) \leq(\lambda+3 \rho) d_{C^{0}}(\bar{x}, \bar{y})$.

If $x \in W_{l o c}^{u}(y)$ then $d_{C^{0}}\left(\mathfrak{g}^{-1} \bar{x}, \mathfrak{g}^{-1} \bar{y}\right) \leq(\lambda+3 \rho) d_{C^{0}}(\bar{x}, \bar{y})$.

Proof of Lemma 3.1. Let us prove the first part of Lemma. The proof of the second part is similar.

If $x \in W_{l o c}^{s}(y)$, then for any point $(\hat{x}(\varphi), \varphi)$ a strong stable manifold $W_{l o c}^{s s}(\hat{x}(\varphi), \varphi)$ contains a point $\left(\hat{y}\left(\varphi_{1}\right), \varphi_{1}\right) \in \bar{y}$. Let us define a distance along strong stable manifold between the points $(\hat{x}(\varphi), \varphi)$ and $\left(\hat{y}\left(\varphi_{1}\right), \varphi_{1}\right)$ as infinum of lengths of smooth curves which connect the points $(\hat{x}(\varphi), \varphi)$ and $\left(\hat{y}\left(\varphi_{1}\right), \varphi_{1}\right)$ and are contained in $W_{l o c}^{s s}(\hat{x}(\varphi), \varphi)$. Now we can define the distance $\tilde{d}_{C^{0}}(\bar{x}, \bar{y})$ as maximum of distances along strong stable manifolds between points $(\hat{x}(\varphi), \varphi)$ and $W_{l o c}^{s s}(\hat{x}(\varphi), \varphi) \cap \bar{y}$ over all the points of the leaf $\bar{x}$.

Proposition 3.1. If $x \in W_{l o c}^{s}(y)$ then $\tilde{d}_{C^{0}}(\mathfrak{g} \bar{x}, \mathfrak{g} \bar{y}) \leq(\lambda+2 \rho) \tilde{d}_{C^{0}}(\bar{x}, \bar{y})$.

Proof of Proposition 3.1. Consider points $(\hat{x}(\varphi), \varphi) \in \bar{x}$ and $\left(\hat{y}\left(\varphi_{1}\right), \varphi_{1}\right) \in \bar{y}$ such that $\left(\hat{y}\left(\varphi_{1}\right), \varphi_{1}\right) \in W_{l o c}^{s s}(\hat{x}(\varphi), \varphi)$ and the distance between $\mathfrak{G}(\hat{x}(\varphi), \varphi)$ and $\mathfrak{G}\left(\hat{y}\left(\varphi_{1}\right), \varphi_{1}\right)$ along strong stable manifold is equal to $\tilde{d}_{C^{0}}(\mathfrak{g} \bar{x}, \mathfrak{g} \bar{y})$. Let $\gamma^{s s}$ be a smooth curve connecting the points $(\hat{x}(\varphi), \varphi)$ and $\left(\hat{y}\left(\varphi_{1}\right), \varphi_{1}\right)$, which is contained in $W_{l o c}^{s s}(\hat{x}(\varphi), \varphi)$ and has a length $\left|\gamma^{s s}\right|$ equal to the length between $(\hat{x}(\varphi), \varphi)$ and $\left(\hat{y}\left(\varphi_{1}\right), \varphi_{1}\right)$ along strong stable manifold. Restriction of the map $\mathfrak{F}$ to a strong stable manifold contracts with the coefficient less than $(\lambda+2 \rho)$ (if $x$ and $y$ are close enough). If the map $\mathfrak{G}$ is $C^{1}$-close to $\mathfrak{F}$, then a restriction of $\mathfrak{G}$ to its strong stable manifold contracts with coefficient at most $(\lambda+2 \rho)$. Therefore $\left|\mathfrak{G}\left(\gamma^{s s}\right)\right| \leq(\lambda+2 \rho)\left|\gamma^{s s}\right|$. Note that the distance between $\mathfrak{G}(\hat{x}(\varphi), \varphi)$ and $\mathfrak{G}\left(\hat{y}\left(\varphi_{1}\right), \varphi_{1}\right)$ along a strong stable manifold is not greater than $\left|\mathfrak{G}\left(\gamma^{s s}\right)\right|$. Finally we have $\tilde{d}_{C^{0}}(\mathfrak{g} \bar{x}, \mathfrak{g} \bar{y}) \leq\left|\mathfrak{G}\left(\gamma^{s s}\right)\right| \leq(\lambda+2 \rho)\left|\gamma^{s s}\right| \leq$ $(\lambda+2 \rho) \tilde{d}_{C^{0}}(\bar{x}, \bar{y})$. Proposition 3.1 is proved. 
Proposition 3.2. For any $\alpha>0$ there exist a $C^{1}$-neighborhood of the map $\mathfrak{F}$ and $\varepsilon>0$ such that for any $\mathfrak{G}$ from this neighborhood and any $x, y$ such that $x \in W_{\varepsilon}^{s}(y)$ the following inequality holds

$$
(1-\alpha) \tilde{d}_{C^{0}}(\bar{x}, \bar{y}) \leq d_{C^{0}}(\bar{x}, \bar{y}) \leq(1+\alpha) \tilde{d}_{C^{0}}(\bar{x}, \bar{y}) .
$$

Proof of Proposition 3.2. If $v \in T_{(x, \varphi)}(N \times M)=T_{x} N \oplus T_{\varphi}$ then $v=v^{b}+v^{c}, v^{b} \in$ $T_{x} N, v^{c} \in T_{\varphi} M$. Let us define $\theta$-cones

$$
K^{b}=\left\{v \mid \theta\left\|v^{b}\right\|>\left\|v^{c}\right\|\right\}, K^{c}=\left\{v \mid \theta\left\|v^{c}\right\|>\left\|v^{b}\right\|\right\}
$$

If $v$ is a vector from a tangent space to a central leaf $\bar{x}$ of the map $\mathfrak{F}$ at a point $(x, \varphi)) \in \bar{x}$ then $v \in K_{(x, \varphi)}^{c}$.

If $v$ is a vector from a tangent space to a strong stable manifold $W_{l o c}^{s s}(x, \varphi)$ at a point $\left(x_{1}, \varphi_{1}\right) \in W_{l o c}^{s s}(x, \varphi)$ then $v \in K_{\left(x_{1}, \varphi_{1}\right)}^{b}$.

The same holds for the map $\mathfrak{G}$ if $\mathfrak{G}$ is $C^{1}$-close to $\mathfrak{F}$, because central leaves (strong stable manifolds) of the map $\mathfrak{G}$ are $C^{1}$-close to the central leaves (strong stable manifolds) of the map $\mathfrak{F}$.

Let $(\hat{x}(\varphi), \varphi) \in \bar{x},\left(\hat{y}\left(\varphi_{1}\right), \varphi_{1}\right) \in \bar{y}, \quad\left(\hat{y}\left(\varphi_{1}\right), \varphi_{1}\right) \in W_{l o c}^{s s}(\hat{x}(\varphi), \varphi)$.

Let $\gamma^{b}$ be a curve in $N$ which connects points $x$ and $y$ and realizes the distance between them:

$$
d=d_{N}(x, y)=\left|\gamma^{b}\right|
$$

Let $\gamma^{s s}$ be a curve in $W_{l o c}^{s s}(x, \varphi)$, which connects points $(x, \varphi)$ and $\left(y_{1}, \varphi_{1}\right)$ and realizes the distance (along the strong stable manifold) between them:

$$
\tilde{d}=d_{\text {along } W_{l o c}^{s s}(x, \varphi)}\left((x, \varphi),\left(y_{1}, \varphi_{1}\right)\right)=\left|\gamma^{s s}\right| .
$$

Let $c=d_{M}\left(\varphi, \varphi_{1}\right)$, and a curve $l \subset M$ connects $\varphi$ and $\varphi_{1}$ and realizes the distance between them:

$$
c=d_{M}\left(\varphi, \varphi_{1}\right)=|l| .
$$

Finally, let $\gamma^{c}$ be a lifting of the curve $l$ to the leaf $\hat{y}$, that is $\gamma^{c}$ connects the points $(y, \varphi)$ and $\left(y_{1}, \varphi_{1}\right)$, and a projection $\gamma^{c}$ to $M$ coinside with $l$. Let us denote by $\gamma^{\Delta}$ a projection $\gamma^{c}$ to $N$.

Since a tangent vector to the curve $\gamma^{c}$ at every its point belongs to $K^{c}$, we have

$$
\left|\gamma^{\Delta}\right| \leq \theta|l|=\theta c
$$

Let us denote by $p_{M}(\cdot)$ a projection to $M$, and by $p_{N}(\cdot)$ a projection to $N$. Since a tangent vector to the curve $\gamma^{s s}$ at every point belongs to $K^{b}$, we have

$$
\left|p_{M}\left(\gamma^{s s}\right)\right| \leq \theta\left|p_{N}\left(\gamma^{s s}\right)\right|
$$


Since $c=d_{M}\left(\varphi, \varphi_{1}\right) \leq\left|p_{M}\left(\gamma^{s s}\right)\right|$, we have

$$
\begin{aligned}
d=\left|\gamma^{b}\right| & \leq\left|\gamma^{c}\right|+\left|\gamma^{s s}\right| \leq\left|\gamma^{\Delta}\right|+|l|+\left|\gamma^{s s}\right| \leq \theta c+c+\left|\gamma^{s s} s\right| \leq(1+\theta)\left|p_{M}\left(\gamma^{s s}\right)\right|+\left|\gamma^{s s}\right| \leq \\
& \leq(1+\theta) \theta\left|p_{N}\left(\gamma^{s s}\right)\right|+\left|\gamma^{s s}\right| \leq(1+(1+\theta) \theta)\left|\gamma^{s s}\right|=(1+(1+\theta) \theta) \tilde{d}
\end{aligned}
$$

Farther, $W_{l o c}^{s s}(x, \varphi)$ is $C^{1}$-smooth. So if $(x, \varphi)$ and $(y, \varphi)$ are close enough (which can be guaranteed by the choice of small $\varepsilon$ ), then

$$
\tilde{d} \leq(1+\theta) d_{N \times M}\left((x, \varphi),\left(y_{1}, \varphi_{1}\right)\right) .
$$

But then we have:

$$
\begin{gathered}
\tilde{d} \leq(1+\theta) d_{N \times M}\left((x, \varphi),\left(y_{1} \varphi_{1}\right)\right) \leq(1+\theta)\left(\left|\gamma^{b}\right|+\left|\gamma^{c}\right|\right) \leq \\
\leq(1+\theta)\left(\left|\gamma^{b}\right|+|l|+\left|\gamma^{\Delta}\right|\right) \leq(1+\theta)(d+c+\theta c) \leq(1+\theta)(d+\theta(1+\theta) \tilde{d}) .
\end{gathered}
$$

Hence,

$$
\frac{1-\theta(1+\theta)^{2}}{1+\theta} \tilde{d} \leq d
$$

Therefore, if $\theta$ and $\varepsilon$ are small enough, then

$$
(1-\alpha) \tilde{d} \leq d \leq(1+\alpha) \tilde{d}
$$

Since this is true for every point $\varphi \in M$, we have

$$
(1-\alpha) \tilde{d}_{C^{0}}(\bar{x}, \bar{y}) \leq d_{C^{0}}(\bar{x}, \bar{y}) \leq(1+\alpha) \tilde{d}_{C^{0}}(\bar{x}, \bar{y})
$$

Proposition 3.2 is proved.

Lemma 3.1 follows now from Propositions 3.1 and 3.2. Indeed, if $x \in W_{l o c}^{s}(y)$, then

$d_{C^{0}}(\mathfrak{g} \bar{x}, \mathfrak{g} \bar{y}) \leq(1+\alpha) \tilde{d}_{C^{0}}(\mathfrak{g} \bar{x}, \mathfrak{g} \bar{y}) \leq(1+\alpha)(\lambda+2 \rho) \tilde{d}_{C^{0}}(\bar{x}, \bar{y}) \leq \frac{1+\alpha}{1-\alpha}(\lambda+2 \rho) d_{C^{0}}(\bar{x}, \bar{y})$

This implies that if $\alpha$ is small enough, then $d_{C^{0}}(\mathfrak{g} \bar{x}, \mathfrak{g} \bar{y}) \leq(\lambda+3 \rho) d_{C^{0}}(\bar{x}, \bar{y})$. Lemma 3.1 is proved.

Now let us show that that the map $\mathfrak{g}: \mathfrak{L}^{0} \rightarrow \mathfrak{L}^{0}$ satisfies the condition (IH1).

Since $\Lambda$ is a locally maximal hyperbolic set of the map $S: N \rightarrow N$, there exist $\varepsilon>\delta>0$ such that the following holds. If $d_{N}(x, y)<\delta$ then there exists the only point $w=w(x, y) \in \Lambda$ such that $\{w\}=W_{\varepsilon}^{u}(x) \cap W_{\varepsilon}^{s}(y)=W_{3 \varepsilon}^{u}(x) \cap W_{3 \varepsilon}^{s}(y)$. Fix small $\rho>0, \rho<<\delta$. If $\mathfrak{G}$ is $C^{1}$-close enough to $\mathfrak{F}$ then $C^{0}$-distance between any central leaf of $\mathfrak{G}$ and the corresponding central leaf of $\mathfrak{F}$ is not greater that $\rho$ (see [13], Theorem 6.8). 
Lemma 3.2. If $\bar{x}$ and $\bar{y}$ are central leaves of the map $\mathfrak{G}, \bar{x}, \bar{y} \in \mathfrak{L}^{0}$, and $d_{C^{0}}(\bar{x}, \bar{y}) \leq$ $\delta-2 \rho$ then there exists the only central leaf $\bar{w} \in \mathfrak{L}^{0}$ of the map $\mathfrak{G}$ such that $\bar{w} \in$ $W_{2 \varepsilon}^{s}(\bar{x}) \cap W_{2 \varepsilon}^{u}(\bar{y})$.

Proof of Lemma 3.2. First let us show that the intersection $W_{2 \varepsilon}^{u}(x) \cap W_{2 \varepsilon}^{s}(y) \subset$ $\mathfrak{L}^{0}$ can not contain more than one central leaf of the map $\mathfrak{G}$. Assume that the intersection $W_{\varepsilon}^{u}(x) \cap W_{\varepsilon}^{s}(y)$ is not empty and take any point $\bar{w} \in \mathfrak{L}^{0}$ from the intersection. Consider the corresponding central leaves $\{x\} \times M,\{y\} \times M$ and $\{w\} \times M$ of the map $\mathfrak{F}$. Here $x=\Phi(\bar{x}), y=\Phi(\bar{y})$ and $w=\Phi(\bar{w})$. By our assumption $d_{C^{0}}(\{x\} \times M, \bar{x})<\rho, d_{C^{0}}(\{y\} \times M, \bar{y})<\rho$ and $d_{C^{0}}(\{w\} \times M, \bar{w})<\rho$. Moreover, for all $\left.\left.n \in \mathbb{Z} d_{C^{0}}\left(\left\{S^{n}(x)\right\} \times M, \mathfrak{g}^{n} \bar{x}\right)<\rho\right), d_{C^{0}}\left(\left\{S^{n}(y)\right\} \times M, \mathfrak{g}^{n} \bar{y}\right)<\rho\right)$ and $\left.d_{C^{0}}\left(\left\{S^{n}(w)\right\} \times M, \mathfrak{g}^{n} \bar{w}\right)<\rho\right)$. Together with Lemma 3.1 this implies that $w \in W_{2 \varepsilon+2 \rho}^{u}(x) \cap W_{2 \varepsilon+2 \rho}^{s}(y)$. Indeed, for all $k \in \mathbb{N}$ we have

$$
\begin{gathered}
d_{N}\left(S^{k}(w), S^{k}(x)\right)=d_{C^{0}}\left(\left\{S^{k}(w)\right\} \times M,\left\{S^{k}(x)\right\} \times M\right) \leq \\
\leq d_{C^{0}}\left(\left\{S^{k}(w)\right\} \times M, \mathfrak{g}^{k} \bar{w}\right)+d_{C^{0}}\left(\mathfrak{g}^{k} \bar{w}, \mathfrak{g}^{k} \bar{x}\right)+d_{C^{0}}\left(\mathfrak{g}^{k} \bar{x},\left\{S^{k}(x)\right\} \times M\right) \leq \\
\leq 2 \rho+d_{C^{0}}(\bar{w}, \bar{x}) \leq 2 \rho+2 \varepsilon,
\end{gathered}
$$

so $w \in W_{2 \varepsilon+2 \rho}^{s}(x)$. Similarly $w \in W_{2 \varepsilon+2 \rho}^{u}(y)$.

Since $d_{C^{0}}(\bar{x}, \bar{y}) \leq \delta-2 \rho$, we have

$$
\begin{gathered}
d_{N}(x, y)=d_{C^{0}}(\{x\} \times M,\{y\} \times M) \leq d_{C^{0}}(\{x\} \times M, \bar{x})+d_{C^{0}}(\bar{x}, \bar{y})+d_{C^{0}}(\bar{y},\{y\} \times M) \leq \\
\leq(\delta-2 \rho)+\rho+\rho=\delta .
\end{gathered}
$$

By our choice of $\delta$ and $\varepsilon$ there exists the only point $w \in W_{3 \varepsilon}^{s}(x) \cap W_{3 \varepsilon}^{u}(y)=$ $W_{\varepsilon}^{s}(x) \cap W_{\varepsilon}^{u}(y)$. Uniqueness is proved.

Now let us prove existence. Since $d_{N}(x, y) \leq \delta$, there exists a point $w \in W_{\varepsilon}^{s}(x) \cap$ $W_{\varepsilon}^{u}(y) \in \Lambda$. Consider the corresponding central leaf $\bar{w}=\Phi^{-1}(w) \in \mathfrak{L}^{0}$ of the map $\mathfrak{G}$. We have for all $k \in \mathbb{Z}^{+}$

$$
\begin{gathered}
d_{C^{0}}\left(\mathfrak{g}^{k} \bar{x}, \mathfrak{g}^{k} \bar{w}\right) \leq d_{C^{0}}\left(\mathfrak{G}^{k} \bar{w},\left\{S^{k}(w)\right\} \times M\right)+ \\
+d_{C^{0}}\left(\left\{S^{k}(w)\right\} \times M,\left\{S^{k}(x)\right\} \times M\right)+d_{C^{0}}\left(\left\{S^{k}(x)\right\} \times M, \mathfrak{g}^{k} \bar{x}\right) \leq 2 \rho+\varepsilon .
\end{gathered}
$$

Therefore $\bar{x} \in W_{\varepsilon+2 \rho}^{s}(\bar{x}) \subset \mathfrak{L}^{0}$. Lemma 3.2 is proved.

Lemma 3.3. The map $\mathfrak{g}: \mathfrak{L}^{0} \rightarrow \mathfrak{L}^{0}$, induced in the space of central leaves of the map $\mathfrak{G}$ with $C^{0}$-metric, is a lipschitzemorphism.

Proof of Lemma 3.3. Set $L=\max _{(x, \varphi) \in N \times M}\|D \mathfrak{G}(x, \varphi)\|$. Let us recall that $C^{0}$ distance between central leaves $\bar{x}$ and $\bar{y}$ was defined by

$$
d_{C^{0}}(\bar{x}, \bar{y})=\max _{\varphi \in M} d_{N}(\hat{x}(\varphi), \hat{y}(\varphi))
$$


Fix $\varphi \in M$. Let $\gamma^{b}$ be a curve in $N \times\{\varphi\} \subset N \times M$ that connects points $(\hat{x}(\varphi), \varphi)$ and $(\hat{y}(\varphi), \varphi)$ and realizes distance between them, $\left|\gamma^{b}\right|=d_{N}(\hat{x}(\varphi), \hat{y}(\varphi))$. Let us denote by $p_{M}(\cdot)$ and $p_{N}(\cdot)$ the projection to $M$ and $N$, respectively. Denote $\varphi_{1}=$ $p_{M}(\mathfrak{G}(\hat{x}(\varphi), \varphi))$ and $\varphi_{2}=p_{M}(\mathfrak{G}(\hat{y}(\varphi), \varphi))$. Then the curve $\mathfrak{G}\left(\gamma^{b}\right)$ connects points $\mathfrak{G}(\hat{x}(\varphi), \varphi)=\left(\widehat{S(x)}\left(\varphi_{1}\right), \varphi_{1}\right)$ and $\mathfrak{G}(\hat{y}(\varphi), \varphi)=\left(\widehat{S(y)}\left(\varphi_{2}\right), \varphi_{2}\right)$.

Denote by $\gamma^{c}$ the lifting of the curve $p_{M}\left(\mathfrak{G}\left(\gamma^{b}\right)\right) \subset M$ to the leaf $\overline{S(y)}=\mathfrak{g} \bar{y}$, $\gamma^{c} \subset \mathfrak{g} \bar{y}, p_{M}\left(\gamma^{c}\right)=p_{M}\left(\mathfrak{G}\left(\gamma^{b}\right)\right)$, and $\gamma^{c}$ connects points $\left(\widehat{S(y)}\left(\varphi_{1}\right), \varphi_{1}\right) \in \mathfrak{g} \bar{y}$ and $\left(\widehat{S(y)}\left(\varphi_{2}\right), \varphi_{2}\right) \in \mathfrak{g} \bar{y}$. By triangle inequality we have

$$
d_{N}\left(\widehat{S(x)}\left(\varphi_{1}\right), \widehat{S(y)}\left(\varphi_{1}\right)\right) \leq\left|\gamma^{c}\right|+\left|\mathfrak{G}\left(\gamma^{b}\right)\right|
$$

Define $\theta$-cones $K^{b}$ and $K^{c}$ by (3.4).

If $\mathfrak{G}$ is $C^{1}$-close to $\mathfrak{F}$, tangent vector to the curve $\mathfrak{G}\left(\gamma^{b}\right)$ belongs to $K^{b}$ at each point. This implies that

$$
\left|p_{M}\left(\mathfrak{G}\left(\gamma^{b}\right)\right)\right| \leq \theta\left|p_{N}\left(\mathfrak{G}\left(\gamma^{b}\right)\right)\right| \leq \theta\left|\mathfrak{G}\left(\gamma^{b}\right)\right| \leq \theta L\left|\gamma^{b}\right|
$$

Since central leaves of $\mathfrak{G}$ are $C^{1}$-close to central leaves of $\mathfrak{F}$, in particular, tangent vector to the curve $\gamma^{c}$ belongs to $K^{c}$ at each point, we have

$$
\left|p_{N}\left(\gamma^{c}\right)\right| \leq \theta\left|p_{M}\left(\gamma^{c}\right)\right|=\theta\left|p_{M}\left(\mathfrak{G}\left(\gamma^{b}\right)\right)\right| \leq \theta\left|\mathfrak{G}\left(\gamma^{b}\right)\right| \leq \theta L\left|\gamma^{b}\right|
$$

Finally we have

$$
\begin{gathered}
d_{N}\left(\widehat{S(x)}\left(\varphi_{1}\right), \widehat{S(y)}\left(\varphi_{1}\right)\right) \leq\left|\gamma^{c}\right|+\left|\mathfrak{G}\left(\gamma^{b}\right)\right| \leq\left|p_{N}\left(\gamma^{c}\right)\right|+\left|p_{M}\left(\gamma^{c}\right)\right|+\left|\mathfrak{G}\left(\gamma^{b}\right)\right| \leq \\
\leq \theta L\left|\gamma^{b}\right|+L\left|\gamma^{b}\right|+L\left|\gamma^{b}\right|=(2+\theta) L\left|\gamma^{b}\right|=(2+\theta) L d_{N}(\hat{x}(\varphi), \hat{y}(\varphi)) .
\end{gathered}
$$

Since this inequality holds for all $\varphi \in M$, we have

$$
d_{C^{0}}(\mathfrak{g} \bar{x}, \mathfrak{g} \bar{y}) \leq(2+\theta) L d_{C^{0}}(\bar{x}, \bar{y})
$$

Note that since $L=L(\mathfrak{G})=\max _{(x, \varphi) \in N \times M}\|D \mathfrak{G}(x, \varphi)\|$ is uniformly bounded above for all $\mathfrak{G}$ from a small $C^{1}$-neighborhood of $\mathfrak{F}$, the Lipschitz constant for $\mathfrak{g}$ can be chosen uniformly for all $\mathfrak{G}$ near $\mathfrak{F}$.

In the same way one can prove that the map $\mathfrak{g}^{-1}: \mathfrak{L}^{0} \rightarrow \mathfrak{L}^{0}$ is Lipschitz. Lemma 3.3 is proved.

Lemma 3.4. The systems $\left(\Lambda,\left.S\right|_{\Lambda}\right)$ and $\left(\mathfrak{L}^{0}, \mathfrak{g}\right)$ are topologically conjugate.

Proof of Lemma 3.4. The central leaves were defined as preimages $\bar{x}=\Phi^{-1}(x)$, $x \in \Lambda$. Since $\Delta=\cup_{x \in \Lambda} \Phi^{-1}(x)$, the map $\Phi$ induces a map $\bar{\Phi}: \mathfrak{L}^{0} \rightarrow \Lambda, \bar{\Phi}(\bar{x})=x$, 
on the space of central leaves. The map is a semiconjugacy, i.e. the following diagram commutes:

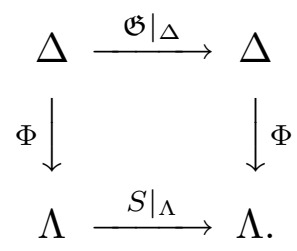

Therefore, the diagram

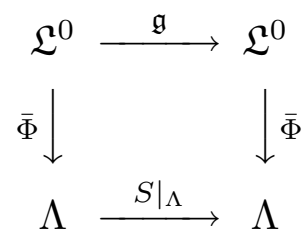

commutes too. So to prove that $\bar{\Phi}$ is a conjugacy we just need to check that $\bar{\Phi}$ is a homeomorphism. Since the map $\Phi: \Delta \rightarrow \Lambda$ is continuous, the map $\bar{\Phi}: \mathfrak{L}^{0} \rightarrow \Lambda$ is also continuous. We need only to show that the map $\bar{\Phi}^{-1}: \Lambda \rightarrow \mathfrak{L}^{0}$ is continuous.

Assume that it is not true, and there exists a sequence of points $\left\{x_{n}\right\} \subset \Lambda$ and a point $y \in \Lambda$ such that $x_{n} \rightarrow y$ in $\Lambda$ but $\bar{x}_{n} \not \supset \bar{y}$ in $\mathfrak{L}^{0}$. Taking a subsequence if necessary, we can assume that for some $\xi>0$ and all $n \in \mathbb{N}$ we have $d_{C^{0}}\left(\bar{x}_{n}, \bar{y}\right)>\xi$.

Let $\varepsilon, \delta$ and $\rho$ be as in Lemma 3.2.

Proposition 3.3. There exists $l \in \mathbb{N}, l=l(\xi, \rho, \mathfrak{G})$, such that if $d_{C^{0}}(\bar{x}, \bar{y})>\xi$ then $d_{C^{0}}\left(\mathfrak{g}^{k} \bar{x}, \mathfrak{g}^{k} \bar{y}\right)>3 \rho$ for some $|k| \leq l$.

Proof of Proposition 3.3. Assume that $d_{C^{0}}(\bar{x}, \bar{y})<3 \rho \ll \delta<\varepsilon$ (otherwise there is nothing to prove). By Lemma 3.2 there exists a point $\bar{z} \in \mathfrak{L}^{0}$ such that $\bar{z}=$ $W_{2 \varepsilon}^{s}(\bar{x}) \cap W_{2 \varepsilon}^{u}(\bar{y})$. Assume that $d_{C^{0}}(\bar{y}, \bar{z}) \geq d_{C^{0}}(\bar{x}, \bar{z})$ (otherwise we consider negative iterates of $\left.\mathfrak{g}: \mathfrak{L}^{0} \rightarrow \mathfrak{L}^{0}\right)$. In particular, this implies that $d_{C^{0}}(\bar{y}, \bar{z}) \geq \frac{1}{2} \xi$.

By Lemma 3.1

$$
d_{C^{0}}\left(\mathfrak{g}^{k} \bar{z}, \mathfrak{g}^{k} \bar{y}\right) \geq(\lambda+3 \rho)^{-k} d_{C^{0}}(\bar{z}, \bar{y}) \geq \frac{1}{2} \xi(\lambda+3 \rho)^{-k}
$$

if $d_{C^{0}}\left(\mathfrak{g}^{i} \bar{z}, \mathfrak{g}^{i} \bar{y}\right)<\varepsilon$ for $i=0,1, \ldots, k-1$, and

$$
d_{C^{0}}\left(\mathfrak{g}^{k} \bar{z}, \mathfrak{g}^{k} \bar{x}\right) \leq(\lambda+3 \rho)^{k} d_{C^{0}}(\bar{z}, \bar{x})
$$

for all $k \in \mathbb{N}$.

There are two possibilities. Either $2 \varepsilon>d_{C^{0}}(\bar{z}, \bar{y})>\frac{6 \rho \lambda}{1-\lambda^{2}}$, or $d_{C^{0}}(\bar{z}, \bar{y}) \leq \frac{6 \rho \lambda}{1-\lambda^{2}} \ll$ $2 \varepsilon$ (we will take $\rho$ small enough, so that $\frac{6 \rho \lambda}{1-\lambda^{2}} \ll \delta<\varepsilon$ ).

In the first case $d_{C^{0}}(\bar{x}, \bar{z}) \leq d_{C^{0}}(\bar{z}, \bar{y})+3 \rho$, and $d_{C^{0}}(\mathfrak{g} \bar{y}, \mathfrak{g} \bar{z}) \geq(\lambda+3 \rho)^{-1} d_{C^{0}}(\bar{y}, \bar{z})$, $d_{C^{0}}(\mathfrak{g} \bar{x}, \mathfrak{g} \bar{z}) \leq(\lambda+3 \rho) d_{C^{0}}(\bar{x}, \bar{z}) \leq(\lambda+3 \rho)\left(3 \rho+d_{C^{0}}(\bar{z}, \bar{y})\right)$. Therefore,

$d_{C^{0}}(\mathfrak{g} \bar{x}, \mathfrak{g} \bar{y}) \geq d_{C^{0}}(\mathfrak{g} \bar{y}, \mathfrak{g} \bar{z})-d_{C^{0}}(\mathfrak{g} \bar{z}, \mathfrak{g} \bar{x}) \geq \frac{1}{\lambda+3 \rho} d_{C^{0}}(\bar{y}, \bar{z})-(\lambda+3 \rho)\left(3 \rho+d_{C^{0}}(\bar{z}, \bar{y})\right)=$ 


$$
\begin{gathered}
=\left(\frac{1}{\lambda+3 \rho}-(\lambda+3 \rho)\right) d_{C^{0}}(\bar{z}, \bar{y})-3 \rho(\lambda+3 \rho)=\frac{1-(\lambda+3 \rho)^{2}}{\lambda+3 \rho} d_{C^{0}}(\bar{y}, \bar{z})-3 \rho(\lambda+3 \rho)> \\
>\frac{\left(1-(\lambda+3 \rho)^{2}\right) 6 \rho \lambda}{(\lambda+3 \rho)\left(1-\lambda^{2}\right)}-3 \rho(\lambda+3 \rho) \geq \frac{6 \rho \lambda}{\lambda+3 \rho}-3 \rho(\lambda+3 \rho) \geq 6 \rho-3 \rho=3 \rho .
\end{gathered}
$$

In the second case

$$
d_{C^{0}}(\bar{z}, \bar{x}) \leq d_{C^{0}}(\bar{z}, \bar{y}) \leq \frac{6 \rho \lambda}{1-\lambda^{2}} \ll 2 \varepsilon
$$

Take the smallest $k \in \mathbb{N}$ such that $\frac{\varepsilon}{\lambda+3 \rho} \leq d_{C^{0}}\left(\mathfrak{g}^{k} \bar{z}, \mathfrak{g}^{k} \bar{y}\right)<\varepsilon$. Since

$$
d_{C^{0}}\left(\mathfrak{g}^{k} \bar{z}, \mathfrak{g}^{k} \bar{y}\right) \geq(\lambda+3 \rho)^{-k} d_{C^{0}}(\bar{z}, \bar{y}) \geq \frac{1}{2} \xi(\lambda+3 \rho)^{-k}
$$

we have the estimate $\frac{1}{2} \xi(\lambda+3 \rho)^{-k} \leq \varepsilon$, so $|k| \leq l=l(\xi, \rho, \mathfrak{G})$.

Since $d_{C^{0}}\left(\mathfrak{g}^{k} \bar{z}, \mathfrak{g}^{k} \bar{x}\right)<d_{C^{0}}(\bar{x}, \bar{z}) \leq \frac{6 \rho \lambda}{1-\lambda^{2}} \ll \delta<\varepsilon$, we have

$$
d_{C^{0}}\left(\mathfrak{g}^{k} \bar{x}, \mathfrak{g}^{k} \bar{y}\right) \geq d_{C^{0}}\left(\mathfrak{g}^{k} \bar{z}, \mathfrak{g}^{k} \bar{y}\right)-d_{C^{0}}\left(\mathfrak{g}^{k} \bar{z}, \mathfrak{g}^{k} \bar{x}\right) \geq \frac{\varepsilon}{\lambda+3 \rho}-\frac{6 \rho \lambda}{1-\lambda^{2}}>3 \rho,
$$

if $\rho$ is small enough.

Proposition 3.3 is proved.

Let us apply Proposition 3.3 to $\bar{x}_{n}$ and $\bar{y}$ for each $n \in \mathbb{N}$. Taking subsequence we can assume that for some $k \in \mathbb{Z}$ and for all $n \in \mathbb{N}$ we have $d_{C^{0}}\left(\mathfrak{g}^{k} \bar{x}_{n}, \mathfrak{g}^{k} \bar{y}\right)>3 \rho$. Since $x_{n} \rightarrow y$ in $\Lambda, S^{k}\left(x_{n}\right) \rightarrow S^{k}(y)$ in $\Lambda$ as $n \rightarrow \infty$. Hence, $d_{C^{0}}\left(\left\{S^{k}\left(x_{n}\right)\right\} \times\right.$ $\left.M,\left\{S^{k}(y)\right\} \times M\right) \rightarrow 0$ as $n \rightarrow \infty$. We know that $d_{C^{0}}\left(\mathfrak{g}^{k} \bar{x}_{n},\left\{S^{k}\left(x_{n}\right)\right\} \times M\right)<\rho$ and $d_{C^{0}}\left(\mathfrak{g}^{k} \bar{y},\left\{S^{k}(y)\right\} \times M\right)<\rho$. But this contradicts to the inequality $d_{C^{0}}\left(\mathfrak{g}^{k} \bar{x}_{n}, \mathfrak{g}^{k} \bar{y}\right)>$ $3 \rho$.

Lemma 3.4 is proved.

Lemmas 3.1, 3.2, 3.3 and 3.4 prove Theorem 3.2 for $r=0$.

To define $C^{r}$-metric in the space of central leaves $\mathfrak{L}$, we need some technical preparations.

3.3. Dynamic trivialization. In this section we reproduce (with some little changes which we need) certain arguments by C.Pugh, M.Shub and A.Wilkinson from [21], relating to trivialization of vector bundles with given dynamics in the base of the bundle. In what follows it allows to avoid difficulties concerning nontriviality of stable and unstable distributions and nontriviality of the tangent bundle to the manifold $M$ (central leaf). 
Vector bundle $E$ over a compact base $X$ always has an inverse bundle, that is, a vector bundle $E^{\prime}$ over $X$, for which there exists an automorphism of vector bundles

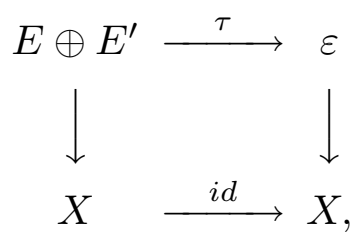

where $\varepsilon$ is a trivial bundle: $\varepsilon=X \times \mathbb{R}^{n}$ for certain $n \in \mathbb{N}$.

In fact a stronger statement holds.

Lemma 3.5 (Dynamic trivialization). (Lemma 3.1 in [21]) Given a vector bundle $E$ over the compact $X$, there exists an inverse bundle $E^{\prime \prime}$ over $X$ such that each vector bundle isomorphism covering a base homeomorphism

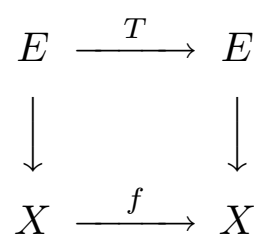

extends to a vector bundle isomorphism

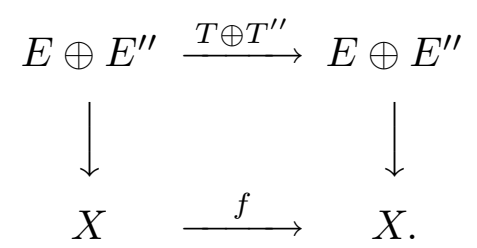

When $E$ and $T$ are smooth, so are $E^{\prime \prime}$ and $T^{\prime \prime}$.

Recall that a function defined on a closed set $\Lambda$ in a manifold $N$ is said to be smooth if it extends to a smooth function defined on a neighborhood of $\Lambda$. Next recall what this means in terms of vector bundles. Let $E$ be a continuous vector bundle over $\Lambda$, where $\Lambda$ is a closed subset of $N$. Then $E$ locally extends to a continuous vector bundle $\widetilde{E}$ over $U$, where $U$ is a neighborhood of $\Lambda$ in $N$. If one such extension $\widetilde{E}$ is smooth, then $E$ itself is said to be smooth. A bundle map $T: E \rightarrow E$ is said to be smooth if it extends to a smooth bundle map of a smooth local extension of $E$. If $E$ is trivial, then $\widetilde{E}$ is also trivial (at least being restricted to a small enough neighborhood of $\Lambda$ ); in this case if $\widetilde{E}$ is a smooth extension, then $\widetilde{E}$ is smoothly trivial.

Therefore, Lemma 3.2 implies that there exists a smooth vector bundle $H^{b}$ over $U(\Lambda)$, which is an inverse bundle for $T_{U(\Lambda)} N$, and $C^{r}$-isomorphism $I^{b}: H^{b} \rightarrow H^{b}$, which covers the map $S$. Fix an inner product structure on $H^{b}$. After multiplying $I^{b}$ by appropriate positive constant, we can assume that $I^{b}$ contracts $H^{b}$ much 
more sharply than $D S$ contracts $E^{s}$. On $U(\Lambda)$ the Lyapunov metric is given, that is, conditions (3.2)and (3.2') hold. Together with the chosen inner product on $H^{b}$, this gives a preferred inner product structure on $T_{U(\Lambda)} N \oplus H^{b}$. The trivial bundle $U(\Lambda) \times \mathbb{R}^{n}$ carries constant Euclidean inner product structure, but the trivializing bundle automorphism

$$
T_{U(\Lambda)} N \oplus H^{b} \cong U(\Lambda) \times \mathbb{R}^{n}
$$

need not to be isometric.

To cope with the lack of isometry, we use a fact from linear algebra. If $<\cdot, \cdot\rangle_{1}$ and $\langle\cdot, \cdot\rangle_{2}$ are inner products on the same finite-dimensional vector space $V$, then there is a canonical isomorphism $Q: V \rightarrow V$ that sends the first inner product to the second, in the sense that for all $v, w \in V$

$$
<Q v, Q w>_{2}=<v, w>_{1} \text {. }
$$

To find $Q$, note that for each $v \in V$, there is unique $v^{\prime} \in V$ such that for all $w \in V$

$$
<v^{\prime}, w>_{2}=<v, w>_{1} .
$$

The mapping $T: v \mapsto v^{\prime}$ is an automorphism of $V$, which is positive definite symmetric with respect to the inner product $<\cdot, \cdot>_{2}$. Set $Q=\sqrt{T}$, where $\sqrt{T}$ is the unique positive definite symmetric square root of $T$. Then, for all $v, w \in V$

$$
<Q v, Q w>_{2}=<Q^{2} v, w>_{2}=<T v, w>_{2}=<v, w>_{1} .
$$

Applying this fact from linear algebra fiber-by-fiber gives bundle isomorphism

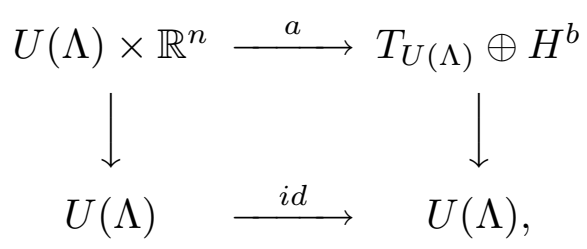

that carry the Euclidean inner product structure to the preferred inner product structure. Since all the inner structures are smooth, so is an automorphism $a$.

Now define $D_{S}$ by commutativity of

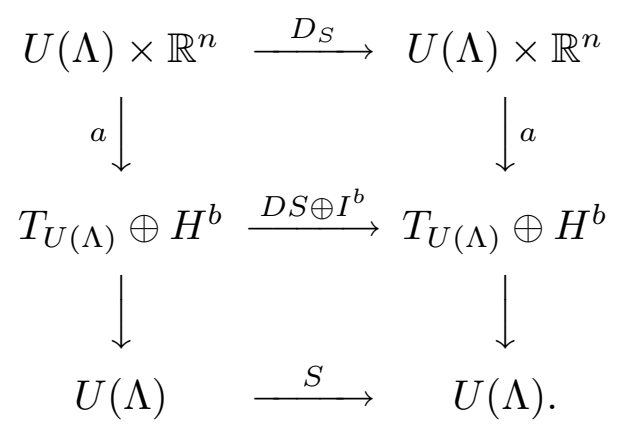

The map $D_{S}: U(\Lambda) \times \mathbb{R}^{n} \hookleftarrow$ is $C^{r}$, has the hyperbolic set $\Lambda$, and a fiber $\mathbb{R}^{n}$ over a point $x \in U(\Lambda)$ is a sum of stable and unstable subspaces $P_{x}^{s}=a^{-1}\left(E_{x}^{s} \oplus H_{x}^{b}\right)$ and $P_{x}^{u}=a^{-1}\left(E_{x}^{u}\right)$. 
3.4. Definition of $C^{r}$-metric in the space of central leaves. We defined a $C^{0}$-metric in the space of central leaves already by (3.1). Now we need to define $C^{r}$-distance between central leaves $\bar{x}$ and $\bar{y}$. These leaves can be considered as graphs of the maps $\hat{x}: M \rightarrow N$ and $\hat{y}: M \rightarrow N$. To define $C^{r}$-distance between $\hat{x}$ and $\hat{y}$, we "lift" them to the space of jets (this is more convenient to us then a usual definition which uses a fixed set of charts). To define the distance in the space of jets we use the trivialization of tangent bundles to $U(\Lambda)$ (as constructed in section 3.3 ) and to $M$.

There exits a smooth bundle $H^{c}$ over $M$, trivializing a tangent bundle $T M$, that is there exists a smooth automorphism $\tau$ of vector bundles:

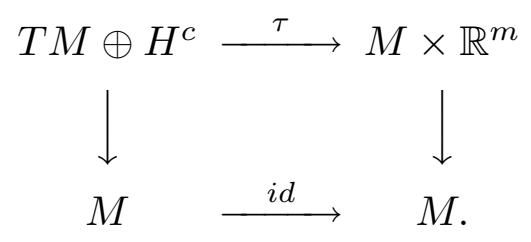

One can assume that the Riemannian metric on $M$ is given in such a way that the isomorphism $T M \oplus H^{c} \cong M \times \mathbb{R}^{m}$ is an isometry.

Consider a central leaf as a graph of the map $\hat{x}: M \rightarrow N$. A tangent space to $\bar{x}$ at the point $(\hat{x}(\varphi), \varphi)$ is a graph of a linear map

$$
L_{\varphi}: T_{\varphi} M \rightarrow T_{\hat{x}(\varphi)} N
$$

Based on $L_{\varphi}$ we can define a linear map

$$
\widetilde{L}_{\varphi}: T_{\varphi} M \oplus H_{\varphi}^{c} \rightarrow T_{\hat{x}(\varphi)} N \oplus H_{\hat{x}(\varphi)}^{b}
$$

in the following way:

$$
T_{\varphi} M \oplus H_{\varphi}^{c} \stackrel{p}{\rightarrow} T_{\varphi} M \stackrel{L_{\varphi}}{\longrightarrow} T_{\hat{x}(\varphi)} N \stackrel{i}{\rightarrow} T_{\hat{x}(\varphi)} N \oplus H_{\hat{x}(\varphi)}^{b}
$$

where $p$ is a projection, $i$ is an inclusion.

Now $\widetilde{L}_{\varphi} \in L\left(\mathbb{R}^{m}, \mathbb{R}^{n}\right)$, where $L\left(\mathbb{R}^{m}, \mathbb{R}^{n}\right)$ is a space of linear maps from $\mathbb{R}^{m}$ to $\mathbb{R}^{n}$. Hence the map $\hat{x}: M \rightarrow U(\Lambda)$ can be lifted to the map $\hat{X}: M \rightarrow U(\Lambda) \times L\left(\mathbb{R}^{m}, \mathbb{R}^{n}\right)$, and we can define a $C^{1}$-distance between central leaves in the following way:

$$
d_{C^{1}}(\bar{x}, \bar{y})=\max _{\varphi \in M} d_{N^{1}}(\hat{X}(\varphi), \hat{Y}(\varphi)), \quad N^{1}=N \times L\left(\mathbb{R}^{m}, \mathbb{R}^{n}\right)
$$

Correspondingly, $C^{r}$-distance between $\bar{x}$ and $\bar{y}$ can be defined inductively, as a $C^{r-1}$-distance between graphs of the maps $\hat{X}$ and $\hat{Y}$. Let us just note, that to trivialize a tangent space to $U(\Lambda) \times L\left(\mathbb{R}^{m}, \mathbb{R}^{n}\right)$ one have to trivialize only a tangent space to $U(\Lambda)$; a linear space $L\left(\mathbb{R}^{m}, \mathbb{R}^{n}\right)$ is already a direct multiplier. 


\subsection{The continuation of a map in the base up to a covering isomor-} phism. Later we will need to continue a small perturbation of a map in the base to a bundle map. In this section we show that such a continuation always exists (Proposition 3.5) .

Proposition 3.4. Let $X$ be a smooth Riemannian manifold, and a map $\mathbf{h}: X \rightarrow X$ be $C^{r}$-close to $i d: X \rightarrow X$. Let $\Pi: E \rightarrow X$ be a vector bundle over $X$ (with the fiber $\mathbb{R}^{k}$ ).

Then there exists an isomorphism of a vector bundle $\mathbf{H}: E \rightarrow E$, covering $\mathbf{h}$ :

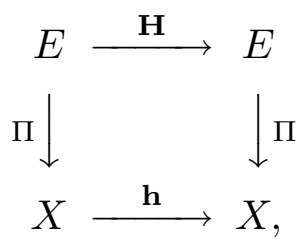

and $C^{r}$-close to id $: E \rightarrow E$.

Proof of the Proposition 3.4. Let us introduce the Riemannian metric on $E$ in the following way. We trivialize a bundle $E$, so it is a subbundle of a trivial bundle $X \times \mathbb{R}^{k}$, consider a standard euclidian structure on $\mathbb{R}^{k}$, the corresponding Riemannian structure on $X \times \mathbb{R}^{k}$, and the induced metric on the bundle $E$. This metric induces a scalar product on each fiber, coincides with the Riemannian metric on $X$, and each fiber is orthogonal to a tangent space to $X$.

Since $\mathbf{h}$ is near identity, there exists the only minimal geodesic from $x$ to $\mathbf{h}(x)$. This arc is also a geodesic in $E$, if one considers $x$ and $\mathbf{h}(x)$ as points in zero section. Indeed, otherwise we can consider a minimal geodesic from $x$ to $\mathbf{h}(x)$; it has to belong to zero section (in another case a projection has a smaller length), but Riemannian metric, induced on a zero section, coincides with the metric on $X$, so we have two different geodesics from $x$ to $\mathbf{h}(x)$ on $X$. Contradiction.

The space of a vector bundle $E$ is a Riemannian manifold. Consider a parallel transport along the constructed geodesic. A tangent space to $X$ will be translated to a tangent space to $X$. A space, orthogonal to $T_{x} X$, will be translated to a space, orthogonal to $T_{\mathbf{h}(x)} X$. Therefore, a fiber over $x$ will be linearly sent to a fiber over $\mathbf{h}(x)$. Constructed map is a required isomorphism $\mathbf{H}: E \rightarrow E$. Proposition 3.4 is proved.

Proposition 3.5. Consider a map $\mathbf{f}: X \rightarrow X, \mathbf{f} \in C^{r}$, and a covering isomorphism $\mathbf{F}$ of a vector bundle $\Pi: E \rightarrow X$ :

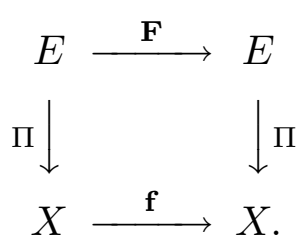

Let the map $\mathbf{g}: X \rightarrow X$ be $C^{r}$-close to $\mathbf{f}$. Then there exists a covering isomorphism of a vector bundle $\mathbf{G}: E \rightarrow E, C^{r}$-close to $\mathbf{F}$ : 


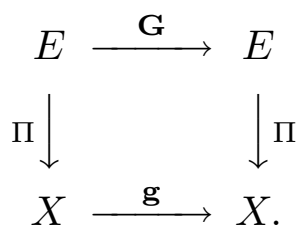

Proof of Proposition 3.5. Consider a map $\mathbf{f}^{-1} \circ \mathbf{g}: X \rightarrow X$. It is $C^{r}$-close to $i d: X \rightarrow X$, hence (due to Proposition 3.4) there exists an isomorphism $\mathbf{H}: E \rightarrow E$, which covers $\mathbf{f}^{-1} \circ \mathbf{g}$ and $C^{r}$-close to $i d: E \rightarrow E$. But in this case the map $\mathbf{F} \circ \mathbf{H}: E \rightarrow E$ is $C^{r}$-close to $\mathbf{F}$ and covers $\mathbf{g}: X \rightarrow X$. Proposition 3.5 is proved.

3.6. Step of induction. We prove Theorem 3.2 by induction. The base of induction (Theorem 3.2 is true for $r=0$ ) is Lemma 3.1. Let us make a step of induction. Assume that Theorem 3.2 holds for $r=r_{0}-1$. Let us show that it holds also for $r=r_{0}>0$.

Tangent bundles to manifolds $N$ and $M$ are trivialized by bundles $H^{b}$ and $H^{c}$ :

$$
T_{U(\Lambda)} N \oplus H^{b} \cong U(\Lambda) \times \mathbb{R}^{n}, T_{M} \oplus H^{c} \cong M \times \mathbb{R}^{m}
$$

Let us denote by $\widetilde{T_{U(\Lambda)} N} \oplus \widetilde{H^{b}}$ a bundle over $U(\Lambda) \times M$ with a fiber $T_{x} N \oplus H_{x}^{b}$ over a point $(x, \varphi)$. Denote by $\widetilde{T M} \oplus \widetilde{H^{c}}$ a bundle over $U(\Lambda) \times M$ with a fiber $T_{\varphi} M \oplus H_{\varphi}^{c}$ over a point $(x, \varphi)$. Therefore a trivial bundle with a fiber $U(\Lambda) \times M$ is given:

$$
\widetilde{T_{U(\Lambda)} N} \oplus \widetilde{H^{b}} \oplus \widetilde{T M} \oplus \widetilde{H^{c}} \cong U(\Lambda) \times M \times \mathbb{R}^{n} \times \mathbb{R}^{m}
$$

A fiber of this bundle at the point $(x, \varphi) \in U(\Lambda) \times M$ is $T_{x} N \oplus H_{x}^{b} \oplus T_{\varphi} M \oplus H_{\varphi}^{b}$.

A map $\mathfrak{F}: U(\Lambda) \times M \hookleftarrow$ has a form $\mathfrak{F}=S \times i d_{M}$. So one can define a map $D_{\mathfrak{F}}: U(\Lambda) \times M \times \mathbb{R}^{n} \times \mathbb{R}^{m} \hookleftarrow$, which covers $\mathfrak{F}$, by $D_{\mathfrak{F}}=D_{S} \oplus i d$. Indeed, the map $D_{S}: U(\Lambda) \times \mathbb{R}^{n} \hookleftarrow$ covers $S: U(M) \hookleftarrow$, and the map $i d: M \times \mathbb{R}^{m} \hookleftarrow$ covers $i d_{M}: M \hookleftarrow$, hence the map $D_{\mathfrak{F}}=D_{S} \oplus i d$ covers the map $\mathfrak{F}=S \times i d_{M}$.

The map $D_{\mathfrak{F}}$, defined in this way, induces the map

$$
\mathfrak{F}^{*}: L\left(\mathbb{R}^{m}, \mathbb{R}^{n}\right) \times U(\Lambda) \times M \hookleftarrow
$$

which covers the map $\mathfrak{F}$. Indeed, a fiber $\mathbb{R}^{m} \times \mathbb{R}^{n}$ over point $(x, \varphi)$ is sent (linearly) by the map $D_{\mathfrak{F}}$ to a fiber $\mathbb{R}^{m} \times \mathbb{R}^{n}$ over point $\mathfrak{F}(x, \varphi)=(S(x), \varphi)$. The graph of a linear map $L: \mathbb{R}_{(x, \varphi)}^{m} \rightarrow \mathbb{R}_{(x, \varphi)}^{M}$ is sent to the graph of a linear map $L^{*}: \mathbb{R}_{\mathfrak{F}(x, \varphi)}^{m} \rightarrow$ $\mathbb{R}_{\widetilde{F}(x, \varphi)}^{M}$. Let us denote $\mathfrak{F}^{*}(L, x, \varphi)=\left(L^{*}, S(x), \varphi\right)$.

Proposition 3.6. The map $\mathfrak{F}^{*}: L\left(\mathbb{R}^{m}, \mathbb{R}^{n}\right) \times U(\Lambda) \times M \hookleftarrow$ has a form $\mathfrak{F}^{*}=$ $\mathfrak{F}_{\text {hyp }}^{*} \times i d_{M}$, where $\mathfrak{F}_{\text {hyp }}^{*}: L\left(\mathbb{R}^{m}, \mathbb{R}^{n}\right) \times U(\Lambda) \hookleftarrow i s$ a hyperbolic map with constant $\lambda$ (that is, with the same constants as a map $S: U(\Lambda) \hookleftarrow$ ).

Proof of Proposition 3.6. The map $D_{S}: U(\Lambda) \times \mathbb{R}^{n} \hookleftarrow$ leaves subbundles $P^{s}$ and $P^{u}$ invariant, $P^{s} \oplus P^{u}=U(\Lambda) \times \mathbb{R}^{n}$, and it contracts fibers $P_{x}^{s}=a^{-1}\left(E_{x}^{s} \oplus H_{x}^{b}\right)$ with 
coefficient not greater than $\lambda$, and expends fibers $P_{x}^{u}=a^{-1}\left(E_{x}^{u}\right)$ with coefficient not less than $\lambda^{-1}$. Therefore, the bundle over $U(\Lambda) \times M$ with the fiber $L\left(\mathbb{R}^{m}, \mathbb{R}^{n}\right)$ is decomposed into the sum of two subbundles, invariant under the action of $\mathfrak{F}^{*}$ :

$$
L_{x}\left(\mathbb{R}^{m}, \mathbb{R}^{n}\right)=L_{x}\left(\mathbb{R}^{m}, P_{x}^{s}\right) \oplus L_{x}\left(\mathbb{R}^{m}, P_{x}^{u}\right)
$$

Moreover, the map $\mathfrak{F}^{*}$ contracts the fibers $L_{x}\left(\mathbb{R}^{m}, P_{x}^{s}\right)$ with coefficient not greater than $\lambda$, and expands the fibers $L_{x}\left(\mathbb{R}^{m}, P_{x}^{u}\right)$ with coefficient not less than $\lambda^{-1}$. Indeed, if $\mathfrak{F}^{*}(L, x, \varphi)=\left(L^{*}, S(x), \varphi\right)$, then $L^{*}=\left(D_{S}\right)_{x} L$, and the above statement is a consequence of that.

Now we just have to note that the action of the map $\mathfrak{F}^{*}$ on a fiber $L\left(\mathbb{R}^{m}, \mathbb{R}^{n}\right)$ over a point $(x, \varphi) \in U(\Lambda) \times M$ depends only on $x \in U(\Lambda)$ and does not depend on $\varphi \in M$. Proposition 3.6 is proved.

If the map $\mathfrak{F}$ has a class of smoothness $C^{r_{0}+1}$, then the map $\mathfrak{F}^{*}$ has a class of smoothness $C^{r_{0}}$, and, therefore, (since, by Proposition 3.6, $\mathfrak{F}^{*}=\mathfrak{F}_{h y p}^{*} \times i d_{M}$ ) Theorem 3.2 (which holds, by induction, for $r=r_{0}-1$ ) can be applied. That is if the map $\mathfrak{G}^{*}$ is $C^{r_{0}}$-close to the map $\mathfrak{F}^{*}$ in a neighborhood of a partially hyperbolic map, then the map, induced in a space of central leaves of the map $\mathfrak{G}^{*}$ with $C^{r_{0}-1}$ metric, is intrinsically hyperbolic with the constant $\lambda^{\prime}>\lambda$ as prescribed in Theorem 3.2 .

Let $\mathfrak{G}: U(\Lambda) \times M$ be $C^{r_{0}+1}$-close to $\mathfrak{F}$. Let us construct a map $\mathfrak{G}^{*}$, such that $\mathfrak{G}^{*}$ is $C^{r_{0}}$-close to $\mathfrak{F}^{*}$ in a neighborhood of the partially hyperbolic set, and central leaves of the map $\mathfrak{G}^{*}$ coincide with the lifts (by (3.3)) of central leaves of the map $\mathfrak{G}$. Since in this case $C^{r_{0}}$-metric in a space of central leaves of the map $\mathfrak{G}$ coincides with $C^{r_{0}-1}$-metric in the space of central leaves of the map $\mathfrak{G}^{*}$, it will prove Theorem 3.2 for $r=r_{0}$.

To construct a required map $\mathfrak{G}^{*}$, let us first construct a bundle map $D_{\mathfrak{G}}: U(\Lambda) \times$ $M \times \mathbb{R}^{n} \times \mathbb{R}^{m} \hookleftarrow$, which is $C^{r_{0}}$-close to the map $D_{\mathfrak{F}}$ and covers the map $\mathfrak{G}$. In order to do this let us recall that

$$
U(\Lambda) \times M \times \mathbb{R}^{n} \times \mathbb{R}^{m} \cong \widetilde{T_{U(\Lambda)} N} \oplus \widetilde{H^{b}} \oplus \widetilde{T M} \oplus \widetilde{H^{c}}
$$

Let us construct a map $D_{\mathfrak{G}}$ as a direct sum of the maps $D \mathfrak{G}: \widetilde{T_{U(\Lambda)} N} \oplus \widetilde{T M} \hookleftarrow$, $D_{\mathfrak{G}}^{b}: \widetilde{H^{b}} \hookleftarrow$ and $D_{\mathfrak{G}}^{c}: \widetilde{H^{c}} \hookleftarrow$, which cover $\mathfrak{G}$. The map $D \mathfrak{G}$ is a differential of the map $\mathfrak{G}$, it is $C^{r_{0}}$-close to the map $D \mathfrak{F}$. A map $D_{\mathfrak{G}}^{b}$ (by Proposition 3.5) can be chosen $C^{r_{0}}$-smooth and $C^{r_{0}}$-close to the map $\widetilde{I}^{b}$, where $\widetilde{I}^{b}$ is a continuation of automorphism $I^{b}: H^{b} \rightarrow H^{b}$ up to the map which covers $\mathfrak{F}$, namely: $\widetilde{I}^{b}\left(H_{(x, \varphi)}^{b}\right)=$ $\left(I^{b}\left(H_{x}^{b}\right)\right)_{(S(x), \varphi)}$. Finally, one can choose a map $D_{\mathfrak{G}}^{c}$ (again by Proposition 3.5 ) to be $C^{r_{0}}$-close to the map $\widetilde{i d}: \widetilde{H^{c}} \rightarrow \widetilde{H^{c}}$, where $\widetilde{i d}$ is a continuation of the map $i d: H^{c} \rightarrow H^{c}$ up to a map which covers $\mathfrak{F}$, namely: $\widetilde{i d}\left(H_{(x, \varphi)}^{c}\right)=H_{(S(x), \varphi)}^{c}$. Then the map $D_{\mathfrak{G}}=D \mathfrak{G} \oplus D_{\mathfrak{G}}^{b} \oplus D_{\mathfrak{G}}^{c}$ has to be $C^{r_{0}}$-close to the map $D_{\mathfrak{F}}=D \mathfrak{F} \oplus \widetilde{I^{b}} \oplus \widetilde{i d}$. 
Let $L_{1}\left(\mathbb{R}^{m}, \mathbb{R}^{n}\right) \subset L\left(\mathbb{R}^{m}, \mathbb{R}^{n}\right)$ be a set of the linear maps with the norm not greater than 1 . Let us define a map $\mathfrak{G}^{*}: L_{1}\left(\mathbb{R}^{m}, \mathbb{R}^{n}\right) \times U(\Lambda) \times M \hookleftarrow$, which covers $\mathfrak{G}: U(\Lambda) \times M \hookleftarrow$, in the following way. If $L \in L_{1}\left(\mathbb{R}^{m}, \mathbb{R}^{n}\right)$, let us set $\mathfrak{G}^{*}(L, x, \varphi)=\left(L^{*}, \mathfrak{G}(x, \varphi)\right)$, where the graph of the map $L^{*}$ is an image of the

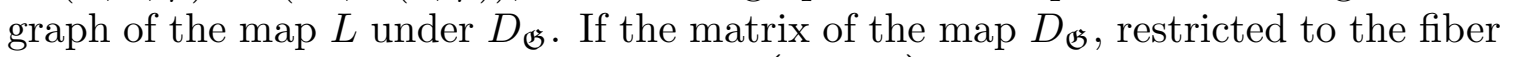
$\mathbb{R}^{m} \times \mathbb{R}^{n}$ over a point $(x, \varphi)$, has a form $\left(\begin{array}{cc}A & B \\ C & D\end{array}\right)$, then

$$
L^{*}=(A L+B)(C L+D)^{-1} .
$$

Since $D_{\mathfrak{G}}$ is near $D_{\mathfrak{F}}$, the matrix of the map $D_{\mathfrak{F}}$ has a form $\left(\begin{array}{cc}\left(D_{S}\right)_{x} & 0 \\ 0 & E\end{array}\right)$, and $L \in L_{1}\left(\mathbb{R}^{m}, \mathbb{R}^{n}\right)$ (i.e. $\|L\| \leq 1$ ), the map $\mathfrak{G}^{*}$ is well defined and $C^{r_{0}}$-close to the restriction of the map $\mathfrak{F}^{*}$ on $L_{1}\left(\mathbb{R}^{m}, \mathbb{R}^{n}\right) \times U(\Lambda) \times M$.

So, we just need to prove the following Proposition.

Proposition 3.7. Central leaves of the map $G^{*}$ coincide with the lifts (by (3.3)) of central leaves of the map $G$.

Proof of Proposition 3.7. The map $\mathfrak{F}^{*}$ has an invariant partially hyperbolic set $\Delta_{\mathfrak{F}}^{*}=\{0\} \times \Lambda \times M$, which is homeomorphic to $\Lambda \times M$ and is locally maximal. The map $\mathfrak{G}^{*}$, since it is $C^{r_{0}}$-close to $\mathfrak{F}^{*}$, also has a partially hyperbolic set $\Delta_{\mathfrak{G}}^{*}$, which is homeomorphic to $\Lambda \times M$, and is locally maximal.

Let us consider a restriction of a bundle over $U(\Lambda) \times M$ with a fiber $L_{1}\left(\mathbb{R}^{m}, \mathbb{R}^{n}\right)$ to the invariant partially hyperbolic set $\Delta_{\mathfrak{G}}$ of the map $\mathfrak{G}$. Denote this restriction

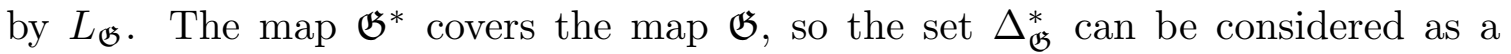
section of the bundle $L_{\mathfrak{G}}$. The set $\Delta_{\mathfrak{G}}^{*}$ is maximal invariant set for $\mathfrak{G}^{*}$, which means that $\Delta_{\mathfrak{G}}^{*}$ is the only section of the bundle $L_{\mathfrak{G}}$ invariant under the action of $\left.\mathfrak{G}^{*}\right|_{L_{\mathfrak{G}}}$. Formula (3.3) defines a map $\Delta_{\mathfrak{G}}$ to $L_{\mathfrak{G}}$. Under this map the operator $L: T_{\varphi} M \rightarrow T_{x} N, \quad(x, \varphi) \in \Delta_{\mathfrak{G}}$, which graph is a tangent space to a central leaf through the point $(x, \varphi)$, is sent to $L^{*}: T_{\varphi} M \oplus H_{\varphi}^{c} \rightarrow T_{x} N \oplus H_{x}^{b}$, where $H_{\varphi}^{c} \subset \operatorname{Ker} L^{*}, \operatorname{Im} L^{*} \subset T_{x} N$. But the tangent spaces to the central leaves of the map $\mathfrak{G}$ are invariant under the action of $D \mathfrak{G}$, and the map $D_{\mathfrak{G}}$ is chosen in such a way that a splitting $T(M \times N) \oplus \widetilde{H^{b}} \oplus \widetilde{H^{c}}$ is invariant. Therefore the section defined by this lifting has to be invariant under $\left.\mathfrak{G}^{*}\right|_{L_{\mathfrak{G}}}$. Invariant section is unique, so it has to coincide with $\Delta_{\mathfrak{G}}^{*}$, that is, the central leaves of the map $\mathfrak{G}^{*}$ coincide with the lifting (by (3.3)) of the central leaves of $\mathfrak{G}$. Proposition 3.7 is proved.

\section{$\S 4$. SOME PROPERTIES OF PARTIALly \\ HYPERBOLIC MAPS: PROOF OF THEOREM A}

Here we prove Theorem A and Addendum.

Note that for the prove it would be enough to provide a section 4.3. Nevertheless we first describe the properties of skew products over Bernoulli shift and their smooth realizations. This construction is a bit easier and has separate applications. 
For example, the systems of this kind appears after bifurcations of saddlenode cycle with several homoclinic surfaces (Theorem B from [9]).

\subsection{Step and mild skew products over Bernoulli shift.}

Here we give some definitions and statements which we will need in Section 4.2. Let $\Sigma^{2}$ be a space of all bi-infinite sequences of 0 and 1, and let $\sigma$ be a Bernoulli shift $\Sigma^{2} \rightarrow \Sigma^{2}$. Metric on $\Sigma^{N}$ can be defined in the following way:

$$
d_{\Sigma^{2}}\left(\omega, \omega^{\prime}\right)=2^{-k}, \quad k=\min \left\{j \in \mathbb{Z}^{+} \mid \omega_{j} \neq \omega_{j}^{\prime} \text { or } \omega_{-j} \neq \omega_{-j}^{\prime}\right\} .
$$

Note that the Bernoulli shift $\sigma: \Sigma^{2} \rightarrow \Sigma^{2}$ is intrinsically hyperbolic, if $\Sigma^{2}$ is equipped with the metric (4.1).

Now consider the map

$$
F: \Sigma^{2} \times S^{1} \rightarrow \Sigma^{2} \times S^{1},(\omega, \varphi) \mapsto\left(\sigma \omega, f_{\omega_{0}}(\varphi)\right)
$$

Note that the map $F$ restricted to a leaf over a point $\omega$ in base depends not on all the sequence $\omega$, but on its element $\omega_{0}$ only. Let us call the skew products of this kind step skew products.

Consider the map

$$
G: \Sigma^{2} \times S^{1} \rightarrow \Sigma^{2} \times S^{1},(\omega, \varphi) \mapsto\left(\sigma \omega, f_{\omega}(\varphi)\right),
$$

where $f_{\omega}: S^{1} \rightarrow S^{1}$ depends on all the sequence $\omega$. Let us call skew products of this kind mild.

In [10] the following Theorem is proved (here we give a bit changed but equivalent statement).

Theorem 4.1. There exist diffeomorphisms $g_{0}, g_{1}: S^{1} \rightarrow S^{1}, g_{0}$ and $g_{1}$ can be taken arbitrary close to id $\in \operatorname{Diff}^{2}\left(S^{1}\right)$, and an interval $I \subset \mathbb{R}, 0 \in I$, such that for all $C>1, \beta>\log _{2} L$, where

$$
L=\max _{i \in\{0,1\}} \max _{\varphi \in S^{1}}\left(\left\|D g_{i}(\varphi)\right\|,\left\|D g_{i}^{-1}(\varphi)\right\|\right),
$$

and small enough neighborhoods $U_{0}\left(g_{0}\right)$ and $U_{1}\left(g_{1}\right)$ in Diff ${ }^{2}\left(S^{1}\right)$ the following holds.

Assume that the map $G$ (4.3) satisfies the conditions:

$$
\begin{gathered}
f_{\omega} \in U_{\omega_{0}} \quad \omega \in \Sigma^{2} \\
d_{C^{1}}\left(f_{\omega}, f_{\omega^{\prime}}\right) \leq C\left(d_{\Sigma^{2}}\left(\omega, \omega^{\prime}\right)\right)^{\beta} \text { for all } \omega, \omega^{\prime} \in \Sigma^{2} .
\end{gathered}
$$

Then the map $G$ has the following properties: 
(i) Periodic orbits of the map $G$ with the multiplier along the circle with absolute value greater than one are dense in $\Sigma^{2} \times S^{1}$. The same holds for periodic orbits with multiplier with absolute value less than one.

(ii) For every $\lambda \in I$ there exists a dense in $\Sigma^{2} \times S^{1}$ orbit with Lyapunov exponent along the circle equal to $\lambda$.

(iii) If an orbit of a sequence $\omega \in \Sigma^{2}$ under the action of Bernoulli shift $\sigma$ is dense in $\Sigma^{2}$, then for every $\varphi \in S^{1}$ an orbit of a point $(\omega, \varphi)$ under the action of $G$ is dense in $\Sigma^{2} \times S^{1}$.

Remark. For step skew products the following statement holds (see [9], [10]). There exists an interval $I \subset \mathbb{R}, 0 \in I$ and open sets $U_{0}, U_{1} \subset \operatorname{Diff}^{1}\left(S^{1}\right)$, such that for all $f_{0}, f_{1}, f_{j} \in U_{j}$ the map $F(4.2)$ has the properties $(i),(i i),(i i i)$, stated in Theorem 4.1 for the $\operatorname{map} G$.

4.2. Smooth realization and the proof of Theorem . Consider a standard Smale horseshoe, realized as a map of two disjoint rectangles. Namely, let $D=$ $D_{0} \cup D_{1}, D^{\prime}=D_{0}^{\prime} \cup D_{1}^{\prime}, \quad D_{0} \cap D_{1}=\varnothing, D_{0}^{\prime} \cap D_{1}^{\prime}=\varnothing$. Let $S: D \rightarrow D^{\prime}$ be a map, such that $\left.S\right|_{D_{i}}: D_{i} \rightarrow D_{i}^{\prime}$ is a restriction to $D_{i}$ of a linear hyperbolic map with constants of contraction and expansion equal to $\nu \in(0,1)$ and $\mu^{-1}>1$, correspondingly. Note that constants $\nu$ and $\mu$ can be chosen arbitrary small by the appropriate choice of rectangles $D$ and $D^{\prime}$.

It is well known that the map $S$ has an invariant set $\Lambda$, homeomorphic to $\Sigma^{2}$, and $\left.S\right|_{\Lambda}$ is conjugated to a Bernoulli shift $\sigma: \Sigma^{2} \rightarrow \Sigma^{2}$.

Consider a locally constant function $i: D \rightarrow\{0,1\}$, defined in the following way: $i(x)=j \Leftrightarrow x \in D_{j}$. A skew product

$$
\mathfrak{F}: D \times S^{1} \rightarrow D^{\prime} \times S^{1}, \mathfrak{F}(x, \varphi)=\left(S(x), f_{i(x)}(\varphi)\right)
$$

is called a smooth realization of a system (4.2).

It is not hard to see that $\left.\mathfrak{F}\right|_{\Lambda \times S^{1}}$ is conjugated to the map $F(4.2)$. The set $\Lambda \times S^{1}$ is partially hyperbolic for $\mathfrak{F}$, and its central leaves are the fibers of the projection to a first factor: $\Lambda \times S^{1} \rightarrow \Lambda, \quad \Lambda \simeq \Sigma^{2}$.

The idea of the proof of Theorem A is the following. Let us take $g_{0}$ and $g_{1}$ as in Theorem 4.1. Consider the map $\mathfrak{F}(4.7)$, which is a smooth realization of $F$ (4.2) with $f_{0}=g_{0}$ and $f_{1}=g_{1}$. If $\mathfrak{G}$ is $C^{2}$-near this map $\mathfrak{F}$, then $\mathfrak{G}$ also has a partially hyperbolic invariant set, which is conjugated to a mild skew product $G$ (4.3). Moreover, the restriction of this conjugacy to any central leaf is a diffeomorphism. If the map $G$ satisfies the conditions of Theorem 4.1, then the map $\mathfrak{G}$ has the properties $(i)$ and $(i i)$ from Theorem A. To be sure that the map $G$ indeed satisfies the conditions of Theorem 4.1, one can do the following.

Let $S$ be a standard Smale horseshoe, $S: D \rightarrow D^{\prime}$. Consider the map $\mathfrak{F}_{0}=$ $S \times i d_{S^{1}}$. Due to Theorem B there exists a $C^{2}$-neighborhood (denote it by $V_{1}$ ) of the map $\mathfrak{F}_{0}$, such that for any map $\mathfrak{G}$ from $V_{1}$ its central leaves depend Hölder continuously on point in $\Lambda$ in $C^{1}$-norm with uniform Hölder exponent and Hölder constant. The systems $\left(\Lambda,\left.S\right|_{\Lambda}\right)$ and $\left(\Sigma^{2}, \sigma\right)$ are conjugated, and a conjugacy is also 
Hölder continuous. Hence, there exist constants $C>1$ and $\beta>0$, such that a restriction of any map $\mathfrak{G} \in V_{1}$ to its invariant partially hyperbolic set is conjugated to a map $G$ of the form (4.3), such that the condition (4.6) holds.

Now, let us define a function $L: V_{1} \rightarrow \mathbb{R}$ in the following way. If a restriction of the map $\mathfrak{G} \in V_{1}$ to its invariant partially hyperbolic set is conjugated to a $\operatorname{map} G(4.3)$, then $L(\mathfrak{G})=\max _{\omega \in \Sigma^{2}} \max _{\varphi \in S^{1}}\left(\left\|D f_{\omega}(\varphi)\right\|,\left\|D f_{\omega}^{-1}(\varphi)\right\|\right)$. Note that $L\left(\mathfrak{F}_{0}\right)=1$. There exists a $C^{2}$-neighborhood of the map $\mathfrak{F}_{0}$ (denote it by $V_{2}$ ), such that $V_{2} \subset V_{1}$, and for any $\mathfrak{G} \in V_{2}$ the value of $L(\mathfrak{G})$ is close to 1 , namely, $\log _{2} L<\beta$.

Now we are prepared to the last step. Due to Theorem 4.1 diffeomorphisms $g_{0}$ and $g_{1}$ can be chosen arbitrary close to $i d \in D i f f^{2}\left(S^{1}\right)$. Take the diffeomorphisms $g_{0}$ and $g_{1}$ in such a way that a smooth realization of the map $F(4.2)$ with $f_{0}=g_{0}$ and $f_{1}=g_{1}$ is in $V_{2}$. Then for any map $\mathfrak{G}$, which is $C^{2}$-close to this smooth realization, its restriction to an invariant partially hyperbolic set is conjugated to a mild skew product $G(4.3)$, and all the conditions of Theorem 4.1 holds (condition (4.6) and inequality $\log _{2} L<\beta, L$ as in (4.4), hold due to the choice of $V_{1}$ and $V_{2}$; condition (4.5) holds, because the central leaves of a perturbed map are $C^{2}$ close to the central leaves of a considered smooth realization). Therefore, $G$ has the properties $(i)$ and $(i i)$ from the statement of Theorem 4.1, hence $\mathfrak{G}$ has the properties $(i)$ and $(i i)$ from the statement of Theorem A. Theorem A is proved.

\subsection{Skew products over solenoid and the proof of Addendum.}

The construction, which proves Addendum, is very similar to the construction from section 4.1 and 4.2. The only difference is that now we have to consider small perturbations of a direct product of a map with hyperbolic attractor (instead of Smale horseshoe) by identity map. Let us take for this purpose a Smale-Williams solenoid: $\mathbf{S}: T \rightarrow T, T=S^{1} \times B, B=\{z \in \mathbb{C}|| z \mid<2\}, S^{1}=\mathbb{R} / \mathbb{Z}, \quad \mathbf{S}(s, z)=$ $\left(2 s, \frac{1}{3} z+\exp 2 \pi i s\right)$. Now consider a skew product

$$
G: T \times S^{1} \rightarrow T \times S^{1},(x, \varphi) \mapsto\left(\mathbf{S}(x), f_{x}(\varphi)\right), x \in T, \varphi \in S^{1} .
$$

For any choice of $f_{x}$, the map (4.8) has a maximal attractor $\Delta$. Let us denote its projection on $T$ along the fibers $S^{1}$ by $\Lambda$. The last set is a solenoid, no matter what $f_{x}$ were chosen.

The analog of Theorem 4.1 for skew products over solenoid is the following Theorem (general approach and a complete proof of the property $(i)$ are given in [10]).

Theorem 4.2. For every $C>1, \beta>0$ one can find $C^{2}$-open sets $U_{0}, U_{1} \subset$ $\operatorname{Diff}^{2}\left(S^{1}\right)$ arbitrary close to id $\in \operatorname{Diff}^{2}\left(S^{1}\right)$, such that the following holds.

Assume that the map $G$ (4.8) has the following properties:

$$
\begin{aligned}
& f_{x} \in U_{0} \quad \text { if } \quad x=(s, z) \in \Lambda_{0}:=\left\{\frac{1}{8} \leq s \leq \frac{1}{2}\right\} ; \\
& f_{x} \in U_{1} \quad \text { if } \quad x=(s, z) \in \Lambda_{1}:=\left\{\frac{5}{8} \leq s \leq 1\right\} ;
\end{aligned}
$$




$$
\begin{gathered}
L=\max _{x \in \Lambda} \max _{\varphi \in S^{1}}\left(\left\|D f_{x}(\varphi)\right\|,\left\|D f_{x}^{-1}(\varphi)\right\|\right), \quad L 2^{-\beta}<1 \\
\left\|D_{\varphi}^{2} f_{x}\right\|<T, \quad\left\|D_{\varphi}^{2} f_{x}^{-1}\right\|<T \quad \text { for some constant } T>0 \text { and all } x, \varphi ; \\
d_{C^{1}}\left(f_{x}, f_{y}\right) \leq C\left(d_{\Lambda}(x, y)\right)^{\beta} \quad \text { for all } x, y \in \Lambda .
\end{gathered}
$$

Then the map $G$ has the following properties:

(i) Periodic orbits of the map $G$ with the multiplier along the circle with absolute value greater than one are dense in $\Lambda \times S^{1}$. The same holds for periodic orbits with multiplier with absolute value less than one.

(ii) There exists an interval $I \subset \mathbb{R}, 0 \in I$, such that for every $\lambda \in I$ there exists a dense in $\Lambda \times S^{1}$ orbit with Lyapunov exponent along the circle equal to $\lambda$.

(iii) If an orbit of a point $x \in \Lambda$ under the action of a map $\mathbf{S}: \Lambda \rightarrow \Lambda$ is dense in $\Lambda$, then for every $\varphi \in S^{1}$ an orbit of a point $(x, \varphi)$ under the action of $G$ is dense in $\Lambda \times S^{1}$.

Now we can make almost the same steps as in section 4.2. Namely, consider a direct product of Smale-Williams solenoid $\mathbf{S}$ by the identity map of a circle. Due to Theorem B there exists a $C^{2}$-neighborhood $W_{1}$ of the map $\mathbf{S} \times i d$, such that any map $\mathfrak{G}$ from $W_{1}$ has a partially hyperbolic map $\Delta$ with $C^{2}$-smooth central leaves, which depend Hölder continuously on a point in base in $C^{1}$-norm. Moreover, Hölder exponent and Hölder constant can be chosen uniformly for all maps $\mathfrak{G}$ from $W_{1}$. It implies that every map $\mathfrak{G} \in W_{1}$ restricted to its invariant partially hyperbolic set $\Delta$ is conjugate to a skew product $G$ (4.8), which satisfies properties (4.12)-(4.13) with uniform constants $\beta>0$ and $C>1$. restriction of this conjugacy to any central leaf is a diffeomorphism. For a fixed $\beta>0$ we can choose another neighborhood $W_{2} \subset W_{1}$, such that for every map $\mathfrak{G} \in W_{2}$ a corresponding skew product satisfies the property (4.11).

Let us take the sets $U_{0}, U_{1} \subset \operatorname{Diff}^{2}\left(S^{1}\right)$ as in Theorem 4.2. If $U_{0}, U_{1}$ are close enough to identity, then there exists an open subset in $W_{2}$, such that for every map $\mathfrak{G}$ from this subset a corresponding skew product satisfies also the properties (4.9) and (4.10). Due to Theorem 4.2 this skew product has the properties $(i)-($ iii) from the statement of Theorem 4.2. It implies that the map $\mathfrak{G}$ has the properties (i), (ii) from the statement of Theorem A. Addendum is proved.

Acknowledgements. Author would like to express an infinite gratitude to his scientific advisor, Yu.Ilyashenko, for a permanent support and unbounded patience. Also I would like to thank A.Wilkinson, M.Shub and D.Dolgopyat for useful discussions and their interest, and Cornell University and personally J.Guckenheimer for hospitality. 


\section{REFERENCES}

1. V.M.Alekseev, M.Yakobson, Symbolic dynamics and hyperbolic dynamical systems, Phys. Rep. 75 (1981), no. 5, 287-325.

2. D.V.Anosov, Geodesic flows on closed Riemann manifolds with negative curvature, Proceedings of the Steklov Institute of Mathematics 90 (1967).

3. C.Bonatti and L.J.Diaz, Nonhyperbolic transitive diffeomorphisms, Ann. of Math. 143 (1996), 357-396.

4. C.Bonatti and M.Viana, SRB measures for partially hyperbolic systems whose central direction is mostly contracting. Preprint IMPA (1997).

5. R.Bowen, Periodic points and measures for Axiom A diffeomorphisms, Trans. Amer. Math. Soc. 154 (1971), 377-397.

6. M.Brin, Hölder continuity of invariant distributions, in Smooth ergodic theory and its applications, A. Katok, R. de la Llave, Ya. Pesin and H. Weiss eds., Proc. Symp. Pure Math., Amer. Math. Soc. (2001).

7. M.Brin, Ya.Pesin, Partially hyperbolic dynamical systems, Izv. Akad. Nauk SSSR Ser. Mat. 38 (1974), 170-212.

8. S.Dawson, C.Grebogi, T.Sauer, J.Yorke, Obstructions to Shadowing When a Lyapunov Exponent Fluctuates about Zero, Physical review letters 73 (1994), no. 14, 1927-1930.

9. A.Gorodetski, Yu.Ilyashenko, Some new robust properties of invariant sets and attractors of dynamical systems, Funktsional. Anal. i Prilozhen 33 (1999), no. 2, 16-30.

10. A.Gorodetski, Yu.Ilyashenko, Some properties of skew products over a horseshoe and a solenoid, Tr. Mat. Inst. Steklova 231 (2000), 96-118.

11. A.Gorodetski, Yu.Ilyashenko, V.Kleptsyn, M.Nalsky, Nonremovability of zero Lyapunov exponents, Funktsional. Anal. i Prilozhen 39 (2005), no. 1, 27-38.

12. B.Hasselblatt, Regularity of the Anosov splitting and of horospheric foliations, Ergodic Theory Dynam. Systems 14 (1994), no. 4, 645-666.

13. M.Hirsh, C.Pugh, M.Shub, Invariant manifolds, Lecture Notes in Math. 583 (1977).

14. Yu.Ilyashenko and W.Li, Nonlocal bifurcations, Amer. Math. Soc., Providence, Long Island (1998).

15. A.Katok and B.Hasselblatt, Introduction to the modern theory of dynamical systems, Cambridge University Press (1995).

16. V.Kleptsyn, M.Nalsky, Stability of existence of non-hyperbolic measures for $C^{1}$-diffeomorphisms, (in preparation).

17. E.J.Kostelich, Ittai Kan, C.Grebogi, E.Ott, J.Yorke, Unstable dimention variability: A source of nonhyperbolicity in chaotic systems, Physica D 109 (1997), 81-90.

18. V.Nitica, A.Török, Cohomology of dynamical systems and rigidity of partially hyperbolic actions of higher-rank lattices, Duke Math. J. 79 (1995), no. 3.

19. V.Nitica, A.Török, An open dense set of stably ergodic diffeomorphisms in a neighborhood of a non-ergodic one, Topology 40 (2001), no. 2, 259-278.

20. Ya.B.Pesin, Lectures on partial hyperbolicity and stable ergodicity, Zürich Lectures in Advanced Mathematics. European Mathematical Society (EMS), Zürich (2004).

21. C.Pugh, M.Shub, A.Wilkinson, Hölder foliations, Duke Math. J. 86 (1997), no. 3, 517-546.

22. D.Ruelle, A.Wilkinson, Absolutely singular dynamical foliations, Comm. Math. Phys. 219 (2001), no. 3, 481-487.

23. J.Schmeling, Ra.Siegmund-Schultze, Hölder continuity of the holonomy maps for hyperbolic basic sets. I, Ergodic theory and related topics, III (Güstrow, 1990), Lecture Notes in Math. 1514 (1992), 174-191.

24. M.Shub, Global Stability of Dynamical Systems, Springer-Verlag, New York (1987).

25. M.Shub, A.Wilkinson, Pathological foliations and removable zero exponents, Invent. Math. 139 (2000), no. 3, 495-508. 
26. G.Ch.Yuan and J.Yorke, An open set of maps for which every point is absolutly nonshadowable, Proc.Amer.Math. 128 (2000), 909-918.

Mathematics 253-37, California Institute of Technology, Pasadena, CA, 91125

E-mail address: asgor@caltech.edu 